\title{
On the Detection of a Scalar Stochastic Background of Gravitational Waves
}

\author{
Danilo Babusci \\ INFN- Laboratori Nazionali di Frascati, I-00044 Frascati, Italy \\ Luca Baiotti \\ SISSA and INFN sez. di Trieste, \\ Via Beirut 4, 34013 Trieste, Italy \\ Francesco Fucito \\ INFN sez. di Roma 2, \\ Via della Ricerca Scientifica, 00133 Roma, Italy \\ Alessandro Nagar \\ Dipartimento di Fisica, Università di Parma \\ and INFN, Gruppo Collegato di Parma, 43100 Parma, Italy
}

\begin{abstract}
In the near future we will witness the coming to a full operational regime of laser interferometers and resonant mass detectors of spherical shape. In this work we study the sensitivity of pairs of such gravitational wave detectors to a scalar stochastic background of gravitational waves. Our computations are carried out both for minimal and non minimal coupling of the scalar fields.
\end{abstract}

\section{INTRODUCTION}

In few years, the research on the detection of gravitational waves (GWs) will hopefully greatly progress. This hope is based on the coming into operation of a new generation of experimental devices that, if they can be operated at the planned sensitivity, should probe deeply into the region in which we believe GWs can be observed. According to the experimental technique employed, these detectors can be divided into two categories: interferometric detectors and resonant mass detectors. To make our point more concrete let us concentrate on Michelson interferometers [1,2] and resonant mass detectors of spherical shape [3]. The main advantage of interferometers is their sensitivity in a wide frequency band. On the other hand spherical shaped resonant mass detectors at resonance have the same sensitivity regardless of the direction of the impinging GW.

In the following we will concentrate on a very specific issue, that is on the possibility of detecting scalar GWs. Our interest in this subject stems from the observation that Einstein's gravity is definitively not the only mathematically consistent theory of gravity and in fact the presence of scalar fields coupled to gravity is required by a vast array of theories that model various phenomena as the inflationary universe or attempt to incorporate gravity with the quantum world. For a review on this subject see Ref. [4]. For more recent proposals that also require a modification of Einstein's gravity see Refs. [5 [7]. Are all the above described detectors fit to measure scalar GWs? While the answer is obvious for resonant mass detector of spherical shape, the situation for interferometers must be analyzed with care. Let us use for a moment the "standard" description, that is well suited for our kind of argument, of an impinging GW (for the moment we neglect its spin content and direction) stretching the lengths $L_{1}$ and $L_{2}$ of the two arms of the interferometer. The conventional Michelson interferometer is configured for maximizing its sensitivity in the detection of the differential mode signal $\Delta_{-}=\delta L_{1}-\delta L_{2}$. Even if the information regarding $L_{1}$ and $L_{2}$ separately is available, the sensitivity of these measurements is orders of magnitude worse than that of $\Delta_{-}$, and, thus, a single interferometer of this type is not able to disentangle the common mode signal $\Delta_{+}=\delta L_{1}+\delta L_{2}$ (transverse monopole mode) from $\Delta_{-}$(usual spin 2 mode). A way out could be the construction of an array of these detectors or the adoption of a different optical configuration (Fox-Smith) for the interferometer [8]. Even if interesting from a theoretical point of view, these alternatives do not seem practical, given the cost and the difficulty in operating such complex apparatus. A viable alternative to these proposals could be, from our point of view, that of a coincidence analysis on the data of an interferometer and a resonant mass detector of spherical shape [9].

In this work we study the sensitivity of combined pairs of resonant mass detectors and interferometers to a scalar stochastic background of gravitational waves (SBGW). If such a background has a flat spectrum (which is the standard assumption) even the narrow frequency band available to a resonant mass detector won't have much influence on our conclusions. Our computations generalize the results of Ref. [9] in which the sensitivity patterns to scalar radiation were considered. To be as general as possible, the impinging radiation is computed in the general setting given by scalar tensor theories [10]. Our main result is the computation of the sensitivity to scalar GWs of correlated pairs of (solid mass or hollow) resonant mass detectors of spherical shape or pairs of interferometer-resonant mass detector. 
Finally we consider the effects on such detectors of massless non minimally coupled scalar fields, generalizing the results of Refs. [11,12]. While this paper was being written, a similar analysis employing two LIGO interferometers for massive and nonrelativistic scalar particles appeared [13].

\section{SCALAR TENSOR THEORY}

\section{A. Fundamental equations}

Let us consider a very general tensor multi scalar theory of gravity, where the gravitational interaction is mediated by $n$ long range scalar fields $\varphi^{a}$ in addition to the usual tensor field present in Einstein's theory. The action in the Einstein frame is

$$
S=(16 \pi G)^{-1} \int \mathrm{d}^{4} x \sqrt{-g}\left(R-2 g^{\mu \nu} \gamma_{a b}\left(\varphi^{c}\right) \partial_{\mu} \varphi^{a} \partial_{\nu} \varphi^{b}\right)+S_{m}\left[\Psi_{m}, A^{2}\left(\varphi^{a}\right) g_{\mu \nu}\right] .
$$

We use units in which the speed of light is $c=1$ and the signature is -+++ . Greek indices $\lambda, \mu, \nu, \ldots=0,1,2,3$ denote spacetime indices; Latin indices from the second part of the alphabet $i, j, k, l \ldots=1,2,3$ denote spatial indices; Latin indices from the first part of the alphabet $a, b, c, \ldots=1, \ldots, n$ label the $n$ scalar fields. Our curvature conventions follow those of Ref. [14]. $R=g^{\mu \nu} R_{\mu \nu}$ is the curvature scalar of the Einstein metric $g_{\mu \nu}$ and $g=\operatorname{det}\left(\mathrm{g}_{\mu \nu}\right)$. The action contains a dimensionful constant $G$, which will be denoted as the bare gravitational constant (related to $\tilde{G}$ Newton's constant as measured by Cavendish experiments) and a $\sigma$ model type metric $\gamma_{a b}(\varphi)$, not necessarily positive definite, in the $n$ dimensional space of the scalar fields. $S_{m}$ denotes the matter action, which is a functional of some matter variables $\Psi_{m}$, and of the Jordan-Fierz metric $\tilde{g}_{\mu \nu} \equiv A^{2}(\varphi) g_{\mu \nu}$. The scalar fields can be non minimally coupled to matter. This means that they can appear as coupling "constants" between the matter fields $\Psi_{m}$ and gravity $\tilde{g}_{\mu \nu}$. For instance, low energy string type theories naturally introduce in the action terms with couplings of the kind

$$
S_{d i l}=-\frac{\beta}{4} \int \mathrm{d}^{4} x \sqrt{-\tilde{g}} \varphi F_{\mu \nu}^{A} F_{\alpha \beta}^{A} \tilde{g}^{\mu \nu} \tilde{g}^{\alpha \beta},
$$

where $F_{\mu \nu}^{A}=\partial_{\mu} A_{\nu}^{A}-\partial_{\nu} A_{\mu}^{A}+f^{A B C} A_{\mu}^{B} A_{\nu}^{C}$ is the Yang-Mills field strength and the scalar field $\varphi$ is the dilaton.

By varying the action $S$ with respect to the Einstein metric $g_{\mu \nu}$ and the scalar fields $\varphi^{a}$, one obtains the following field equations

$$
\begin{array}{r}
R_{\mu \nu}-\frac{1}{2} R g_{\mu \nu}=2 \gamma_{a b}(\varphi)\left(\partial_{\mu} \varphi^{a} \partial_{\nu} \varphi^{b}-\frac{1}{2} g_{\mu \nu} g^{\rho \sigma} \partial_{\rho} \varphi^{a} \partial_{\sigma} \varphi^{b}\right)+8 \pi G T_{\mu \nu} \\
g^{\mu \nu} \nabla_{\mu} \nabla_{\nu} \varphi^{a}+g^{\mu \nu} \gamma_{b c}^{a}(\varphi) \partial_{\mu} \varphi^{b} \partial_{\nu} \varphi^{c}=-4 \pi G\left[\alpha^{a}(\varphi) T+\sigma^{a}\right]
\end{array}
$$

where $\gamma_{b c}^{a}$ are the Christoffel symbols of the metric $\gamma_{a b}(\varphi)$. The functions $\alpha_{a}(\varphi) \equiv \partial_{a} \ln A(\varphi)$ represent the field dependent couplings between scalar fields and matter within the metric sector of the theory. $T^{\mu \nu}=2(-g)^{-1 / 2} \delta S_{m} / \delta g_{\mu \nu}$ is the stress energy tensor, $T$ its trace and $\sigma_{a}=(-g)^{-1 / 2} \delta S_{m} / \delta \varphi^{a}$ is the density of scalar charge. In the Jordan-Fierz frame we would have

$$
T_{\mu \nu}=A^{2}(\varphi) \tilde{T}_{\mu \nu}, \quad \sigma_{a}=A^{4}(\varphi) \tilde{\sigma}_{a},
$$

as can easily be found from their definition [10]. Actually, since $\sqrt{-\tilde{g}}=A^{4}(\varphi) \sqrt{-g}$, and

$$
\delta_{\varphi} S_{m}=\int \mathrm{d}^{4} x \sqrt{-g} \sigma_{a} \delta \varphi^{a}=\int \mathrm{d}^{4} x \sqrt{-\tilde{g}} \tilde{\sigma}_{a} \delta \varphi^{a},
$$

one recovers immediately (2.5).

In the literature, scalar tensor theories with $\sigma_{a}=0$ (i.e. metric theories) have been studied by many authors, from the pioneering work of Jordan, Fierz, Brans, Dicke, Wagoner [15] to the recent studies of Damour and Esposito-Farèse [10]. This interest arises from the fact that they do not violate the Weak Equivalence Principle and so imply geodesic dynamics for neutral weakly self gravitating bodies. However this is not the most general framework, in particular it is not the case of the interesting scalar fields foreseen by string theory. For a recent analysis see Ref. [11.

Let us compute the expression of the relative acceleration between two weakly self gravitating bodies in the general $n$ scalar theory; this formula will be the starting point to write the response of a $\mathrm{GW}$ detector to a scalar tensor wave. 
When $\sigma_{a} \neq 0$, the stress energy conservation law in Einstein units is [10]

$$
\nabla_{\nu} T^{\mu \nu}=\alpha_{a} \nabla^{\mu} \varphi^{a} T-\sigma_{a} \nabla^{\mu} \varphi^{a},
$$

or, in the Jordan-Fierz frame

$$
\tilde{\nabla}^{\nu} \tilde{T}_{\mu \nu}+\tilde{\sigma}_{a} \tilde{\nabla}_{\mu} \varphi^{a}=0
$$

This equation implies a non geodesic motion of test mass bodies. This result corresponds, for a single scalar field and a particular choice of the coupling function $A(\varphi)$, to the lowest order gravidilaton effective action of string theory 111. However, if $\tilde{\sigma}_{a}=0$ we have $\tilde{\nabla}^{\nu} \tilde{T}_{\mu \nu}=0$ and so geodesic motion of test mass bodies is recovered.

In Ref. [11], starting from the single field string like case of (2.8) the equation of motion of test mass bodies has been derived. Following the same line of reasoning, we generalize that result to our case. Let us recall the point like limit of the generally covariant energy momentum tensor for a particle of mass $m$ and world line $x^{\mu}(\tau)$ [14]

$$
\tilde{T}^{\mu \nu}\left(x^{\prime}\right)=\frac{p^{\mu} p^{\nu}}{p^{0} \sqrt{-\tilde{g}}} \delta^{(3)}\left(x^{\prime}-x(\tau)\right),
$$

where $p^{\mu}=m d x^{\mu} / d \tau$. We can rewrite the scalar charge density $\tilde{\sigma}_{a}$ for a test body, in terms of dimensionless scalar functions $\tilde{q}_{a}$, which express the relative strengths of non universal scalar to tensor forces

$$
\tilde{\sigma}_{a}\left(x^{\prime}\right)=-\tilde{q}_{a} \tilde{T}\left(x^{\prime}\right)=\tilde{q}_{a} \frac{m^{2}}{p^{0} \sqrt{-\tilde{g}}} \delta^{(3)}\left(x^{\prime}-x(\tau)\right) .
$$

As we consider long range fields, $\tilde{q}_{a} \ll 1$ to avoid conflicts with the present test of the Weak Equivalence Principle. From (2.8) we get the geodesic equation in scalar tensor theory with non minimal couplings [11]

$$
\ddot{x}^{\mu}+\tilde{\Gamma}_{\alpha \nu}^{\mu} \dot{x}^{\alpha} \dot{x}^{\nu}+\tilde{q}_{a} \partial^{\mu} \varphi^{a}=0,
$$

where $\dot{x}^{\mu} \equiv \mathrm{d} x^{\mu} / \mathrm{d} \tau$. Now we can compute the modifications to the relative acceleration between two test mass bodies moving along two worldlines induced by the $\tilde{q}_{a}$ 's.

Let us take two weakly self gravitating bodies moving along two infinitesimally close worldlines $x^{\mu}(\tau)$ and $x^{\prime \mu}(\tau)=x^{\mu}(\tau)+\delta^{\mu}(\tau)$, where $\delta^{\mu}$ is the separation vector between the two curves. If we suppose that the bodies have different scalar couplings $\tilde{q}_{a}^{(1)}$ and $\tilde{q}_{a}^{(2)}$, their relative acceleration is [14]

$$
\ddot{\delta}_{i}=-\left[\tilde{R}_{i o j o}+\tilde{q}_{a}^{(2)} \partial_{i} \partial_{j} \varphi^{a}\right] \delta_{j}+\left[\tilde{q}_{a}^{(1)}-\tilde{q}_{a}^{(2)}\right] \partial_{i} \varphi^{a},
$$

where $\dot{\delta}_{i} \equiv \mathrm{d} \delta_{i} / \mathrm{d} t$. Notice that in (2.12) there is a term proportional to $\tilde{q}_{a}^{(1)}-\tilde{q}_{a}^{(2)}$. This term will be important when the test mass bodies are of different nature (e.g. one is a baryon and the other one a lepton) but it is irrelevant inside a GW detector. Therefore, the equation needed to analyze the response of GW detectors to scalar tensor waves is

$$
\ddot{\delta}_{i}=-\left[\tilde{R}_{i o j o}+\tilde{q}_{a} \partial_{i} \partial_{j} \varphi^{a}\right] \delta_{j} .
$$

\section{B. Gravitational waves}

Let us recall some results concerning scalar tensor GWs [10]. In the weak field limit of the theory

$$
\begin{aligned}
\tilde{g}_{\mu \nu}(x) & =\eta_{\mu \nu}+\tilde{h}_{\mu \nu}(x), \\
\varphi^{a}(x) & =\varphi_{0}^{a}+\xi^{a}(x),
\end{aligned}
$$

where $\left|\tilde{h}_{\mu \nu}\right| \ll 1,\left|\xi^{a}\right| \ll 1, \eta_{\mu \nu}$ is the flat Minkowski metric, and $\varphi_{0}^{a}$ the background values of the scalar fields. We now choose a gauge in which the metric perturbation has zero time-time and time-space components while the purely spatial components, for a plane wave propagating along the direction characterized by the unit vector $\hat{\Omega}$, assume the form

$$
\tilde{h}_{i j}(x)=h_{A}(x) e_{i j}^{A}(\hat{\Omega})+2 \alpha_{a}^{0} \xi^{a}(x) e_{i j}^{s}(\hat{\Omega}) ; \quad A=+, \times ; \quad a=1, \ldots, n .
$$


$e^{+}, e^{\times}$are the spin 2 polarization tensors describing the ordinary $\mathrm{GW}$ in the transverse traceless gauge, $e^{s}$ is the spin 0 polarization tensor of the scalar waves, $\alpha_{a}^{0} \equiv \alpha_{a}\left(\varphi_{0}^{a}\right)$, and we choose units such that $A\left(\varphi_{0}^{a}\right)=1$. By indicating with $\hat{m}$ and $\hat{n}$ a pair of orthonormal vectors lying in the plane perpendicular to $\hat{\Omega}$, these polarization tensors can be written as follows (see Appendix C2 )

$$
\begin{aligned}
& e_{i j}^{+}(\hat{\Omega})=\hat{m}_{i} \hat{m}_{j}-\hat{n}_{i} \hat{n}_{j}, \\
& e_{i j}^{\times}(\hat{\Omega})=\hat{m}_{i} \hat{n}_{j}+\hat{n}_{i} \hat{m}_{j}, \\
& e_{i j}^{s}(\hat{\Omega})=\delta_{i j}-\hat{\Omega}_{i} \hat{\Omega}_{j}=\hat{m}_{i} \hat{m}_{j}+\hat{n}_{i} \hat{n}_{j},
\end{aligned}
$$

and

$$
e_{i j}^{B}(\hat{\Omega}) e^{B^{\prime} i j}(\hat{\Omega})=2 \delta^{B B^{\prime}} \quad B=+, \times, s .
$$

We consider now the small relative oscillations of two weakly self gravitating bodies induced by this wave. By indicating with $L_{i}$ the rest separation of the bodies, we can put $\delta_{i}=L_{i}+\zeta_{i}\left(\zeta_{i} \ll 1\right)$. Expanding (2.13) to first order in $\zeta_{i}$, we find

$$
\ddot{\zeta}_{i}=-\frac{1}{2}\left[\frac{\mathrm{d}^{2} \tilde{h}_{i j}}{\mathrm{~d} t^{2}}+2 \tilde{q}_{a} \partial_{i} \partial_{j} \xi^{a}\right] L_{j} .
$$

Since we are considering plane wave solutions, the spatial derivatives appearing in the last equation can be replaced by the time derivatives, namely $\partial_{i} \partial_{j} \xi^{a}=\hat{\Omega}_{i} \hat{\Omega}_{j} \ddot{\xi}^{a}=\left(\delta_{i j}-e_{i j}^{s}(\hat{\Omega})\right) \ddot{\xi}^{a}$, and taking into account (2.15), one finds

$$
\ddot{\zeta}_{i}=-\frac{1}{2} \frac{\mathrm{d}^{2}}{\mathrm{~d} t^{2}}\left[h_{A}(x) e_{i j}^{A}(\hat{\Omega})+2\left(\alpha_{a}^{0}-\tilde{q}_{a}\right) \xi^{a}(x) e_{i j}^{s}(\hat{\Omega})+2 \tilde{q}_{a} \xi^{a}(x) \delta_{i j}\right] L_{j},
$$

and then the infinitesimal displacement induced by the GW is

$$
\zeta_{i}=-\frac{1}{2}\left[h_{A}(x) e_{i j}^{A}(\hat{\Omega})+2\left(\alpha_{a}^{0}-\tilde{q}_{a}\right) \xi^{a}(x) e_{i j}^{s}(\hat{\Omega})+2 \tilde{q}_{a} \xi^{a}(x) \delta_{i j}\right] L_{j} .
$$

This formula needs a few comments. The scalar fields considered in our theory are massless, therefore the scalar GW can carry energy and momentum through just one degree of freedom, the transverse polarization tensor $e_{i j}^{s}(\hat{\Omega})$ (see Wagoner in Ref. 115]). Therefore in (2.19) only the transverse part strains the matter and the $\delta_{i j}$ is effectively unimportant when studying the response the antennas to GWs. By introducing the effective gravitational wave sensed by the test mass bodies

$$
\tilde{h}_{i j}^{e f f}=h_{A}(x) e_{i j}^{A}(\hat{\Omega})+2\left(\alpha_{a}^{0}-\tilde{q}_{a}\right) \xi^{a}(x) e_{i j}^{s}(\hat{\Omega})
$$

we rewrite 2.19 ) as follows

$$
\zeta_{i}=-\frac{1}{2} \tilde{h}_{i j}^{e f f} L_{j}
$$

However, if the scalar fields were slightly massive, there would be also a longitudinal polarization along the propagation direction of the GW and we couldn't drop the $\delta_{i j}$ in (2.19). This scenario has been analyzed in [9.11] 13], but in the following we won't consider it and just restrict our study to $\tilde{h}_{i j}^{e f f}$.

\section{INTERFEROMETERS AND RESONANT MASS SPHERICAL DETECTORS}

\section{A. Response function of an interferometer to scalar GWs}

Let us consider a Michelson type laser interferometer with two orthogonal arms of the same nominal length $L_{1}=$ $L_{2}=L$. From (2.21), the signal at the output port of the interferometer (the strain of the differential mode) is proportional to the difference in the two path lengths, $\zeta_{1}-\zeta_{2}$, induced by the wave and can be written in the form 16 


$$
\tilde{h}^{e f f}=\tilde{h}_{i j}^{e f f} \mathcal{D}^{i j},
$$

where $\mathcal{D}$ is a traceless and symmetric tensor describing the geometry of the interferometer 1 . In the interferometer frame, namely the one where the corner station stands at the origin of coordinates and the $\hat{x}$ and $\hat{y}$ axes lie along the arms, this tensor writes

$$
\mathcal{D}=\frac{1}{2}\left(\begin{array}{ccc}
1 & 0 & 0 \\
0 & -1 & 0 \\
0 & 0 & 0
\end{array}\right) .
$$

The effective strain sensed by the interferometer is then split in a spin 2 and a spin 0 part, proportional to the difference $\alpha_{a}^{0}-\tilde{q}_{a}$; we can take explicitly into account the dependence of the strain from the angles $(\theta, \phi)$ defining the direction $\hat{\Omega}$ of the incoming wave by introducing the angular pattern functions of the interferometer

$$
F^{A}(\hat{\Omega})=e_{i j}^{A}(\hat{\Omega}) \mathcal{D}^{i j}, \quad F^{s}(\hat{\Omega})=e_{i j}^{s}(\hat{\Omega}) \mathcal{D}^{i j},
$$

and writing the strain as

$$
\tilde{h}^{e f f}=h_{A}(x) F^{A}(\hat{\Omega})+2\left(\alpha_{a}^{0}-\tilde{q}_{a}\right) \xi^{a}(x) F^{s}(\hat{\Omega}) .
$$

\section{B. Cross section for resonant spheres in scalar tensor theory}

We discuss now the cross section of a resonant sphere in the general scalar tensor theory. For spin 2 waves this result was obtained in Ref. [18] (see also Ref. [19]). In recent years, this kind of detector (both solid and hollow) has been extensively studied as device able to analyze the spin content of GWs (see Refs. [20 22]). The calculation of its scattering cross section in the framework of the Brans-Dicke theory was carried out in Refs. 223 25]. The extension of the results of Ref. [23] to the general scalar tensor theory with minimal coupling is straightforward [26] and in the following subsections 1 and 2, we will just sketch the steps and quote the results. Furthermore, in subsection 3 we will repeat the calculations for the even more general case of $\tilde{q}_{a} \neq 0$. For the sake of generality the direction of propagation of the wave and the antenna frame (defined by a triad of orthonormal vectors $(\hat{x}, \hat{y}, \hat{z})$ ) will be taken to be distinct. The direction $\hat{\Omega}=(\theta, \phi)$ of the incoming wave is identified by the relative orientation of the triad defined in 2.16) with respect to $(\hat{x}, \hat{y}, \hat{z})$

$$
\begin{aligned}
\hat{m} & =\cos \phi \hat{x}+\sin \phi \hat{y}, \\
\hat{n} & =-\sin \phi \cos \theta \hat{x}+\cos \phi \cos \theta \hat{y}+\sin \theta \hat{z}, \\
\hat{\Omega} & =\sin \phi \sin \theta \hat{x}-\cos \phi \sin \theta \hat{y}+\cos \theta \hat{z} .
\end{aligned}
$$

\section{Tensor $G W s$}

Consider a superposition of spin 2 plane GWs with wave vector $k^{\mu}$ and amplitudes $h_{A}$ impinging on a spherical GWs detector

$$
\tilde{h}_{\mu \nu} \equiv \tilde{e}_{\mu \nu} e^{i k_{\rho} x^{\rho}}+\text { c.c. } \equiv h_{A} e_{\mu \nu}^{A} e^{i k_{\rho} x^{\rho}}+\text { c.c. }, \quad A=+, \times .
$$

Note that hereafter $e_{\mu \nu}^{A}$ are the polarization tensors written in the detector frame $(\hat{x}, \hat{y}, \hat{z}$ ) (see Appendix $\mathrm{C} 2$ for their explicit expressions). As usual we will use the so called quadrupole approximation, i.e. we suppose that the detector is much smaller than the wavelength of the impinging GW, so that only the first terms (quadrupole, for the tensor

\footnotetext{
${ }^{1}(3.1)$ is valid in the regime in which the wavelength of the impinging scalar GW is much bigger than the length of the arms of the interferometer. Given the resonant frequencies of our resonant mass detectors, this will be always the case in the present paper. For a more detailed discussion of this point see [17].
} 
component; monopole and quadrupole for the scalar one) have to be considered. Analogously to Ref. [14] we find the expressions for the spin 2 scattering and total energy cross sections

$$
\begin{aligned}
\sigma_{h}^{s c a t} & =\frac{128 \pi G^{2}}{5} \frac{\left[1+\frac{1}{3} \alpha_{0}^{2}\left(1-\alpha_{0}^{2}\right)\right] \tilde{\tau}_{i j}^{*} \tilde{\tau}^{i j}}{\tilde{e}_{i j}^{*} \tilde{e}^{i j}} \\
\sigma_{h}^{t o t} & =\frac{8 G}{f} \frac{\Im\left(\tilde{e}_{i j}^{*} \tilde{\tau}^{i j}\right)}{\tilde{e}_{i j}^{*} \tilde{e}^{i j}},
\end{aligned}
$$

where $\alpha_{0}^{2}=\alpha_{a}^{0} \alpha_{0}^{a}$ and $\tilde{\tau}_{i j} \equiv \tilde{\tau}_{i j}(\hat{\Omega}, f)$ is the (traceless) Fourier transform of the variation induced in the stress energy tensor of the sphere by the impinging GW . Furthermore we will study resonant scattering, i.e. we will assume that the detector scatters only the impinging GWs with frequency $f$ around the resonant frequency of one of its natural vibrational modes. This leads to a relation between $\sigma_{h}^{\text {scat }}$ and $\sigma_{h}^{\text {tot }}$ and to another between $\tilde{\tau}_{i j}$ and the sphere mode tensors

$$
\begin{aligned}
& \sigma_{h}^{s c a t}=\eta \sigma_{h}^{t o t}, \\
& \tilde{\tau}_{i j}=\gamma(f) \tilde{e}_{i j},
\end{aligned}
$$

where $\eta$ is the fraction of the total oscillation energy dissipated through emission of GWs (it can be calculated as a function of the detector internal parameters) and $\gamma(f)$ gives the frequency dependence of $\tilde{\tau}_{i j}$. The function $\gamma(f)$ is chosen so that the response of the antenna is resonant in frequency (see Refs. [20,23]). If the oscillation of the mode with angular momentum $l=2$ has proper frequency $f_{n 2}$ and a bandwidth $\Delta_{f_{n 2}}$, we find

$$
\gamma(f) \propto \frac{1}{f-f_{n 2}+i \Delta_{f_{n 2}} / 2} .
$$

Substituting (3.7) and (3.11) respectively into (3.9) and (3.10) and combining the results, the total energy cross section becomes

$$
\sigma_{h}(f ; n, l=2) \equiv \sigma_{h}^{t o t}(f)=\frac{1}{1+\alpha_{0}^{2}} \frac{\tilde{G} M v^{2} F_{n}}{2 \pi} \frac{\Delta_{f_{n 2}}}{\left(f-f_{n 2}\right)^{2}+\Delta_{f_{n 2}}^{2} / 4},
$$

where $M$ is the sphere mass, $v$ the velocity of sound in the material the sphere is made of, $F_{n}$ a constant depending only on the quadrupolar mode under scrutiny and on the sphere parameters (radius, density, material) [23], and $\tilde{G}=\left(1+\alpha_{0}^{2}\right) G$ is the effective Newton's constant measured in Cavendish like experiments. The results of Ref. 23] in Brans-Dicke theory are recovered by setting $\alpha_{0}^{2}=\left(2 \omega_{B D}+3\right)^{-1}$ where $\omega_{B D}$ is the Brans-Dicke parameter. If in performing this calculation we expand the tensors in the numerator of $\sigma_{h}^{t o t}$ in the detector basis $\mathcal{D}_{i j}^{(\epsilon)}$ (defined in Appendix (C1), we find that the total cross section can be written as the sum of five terms, the total cross sections for any single vibrational mode of the sphere

$$
\sigma_{h}^{t o t}(f)=\sum_{\epsilon} \sigma_{h}^{(\epsilon)}(f, \hat{\Omega}), \quad \epsilon=0,1 c, 1 s, 2 c, 2 s
$$

and

$$
\sigma_{h}^{(\epsilon)}(f, \hat{\Omega})=\frac{1}{1+\alpha_{0}^{2}} \frac{\tilde{G} M v^{2} F_{n}}{2 \pi} \frac{\Delta_{f_{n 2}}}{\left(f-f_{n 2}\right)^{2}+\Delta_{f_{n 2}}^{2} / 4} \frac{\sum_{A}\left|F_{A}^{(\epsilon)} h_{A}\right|^{2}}{\sum_{A^{\prime}}\left|h_{A^{\prime}}\right|^{2}} .
$$

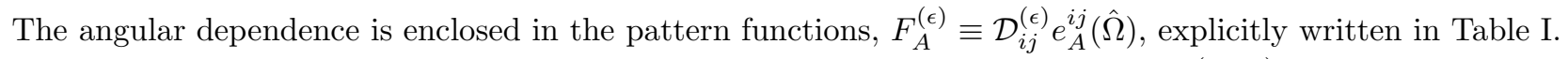

For later purposes we will need also the integrated cross section. By integrating (3.12) we get

$$
\Sigma_{h}(n ; l=2)=\frac{1}{1+\alpha_{0}^{2}} \tilde{G} M v^{2} F_{n} .
$$

\footnotetext{
${ }^{2}$ In principle the expression of $\tilde{\tau}_{i j}$ could contain also a term proportional to $\mathcal{D}_{i j}^{(00)} \propto \delta_{i j}$ [14, accounting for the trace of the polarization tensor (monopole excitation). But, since the trace $\tilde{e}_{i i}$ vanishes, in this tensorial part such a term gives no contribution.

${ }^{3}$ The index $n=1, \ldots, \infty$ labels different solutions for the sphere eigenmodes with fixed angular momentum $l$ 23.
} 


\section{Minimally coupled scalar GWs}

The scattering and total cross section for the minimally coupled scalar part of a GW are

$$
\begin{aligned}
\sigma_{s}^{s c a t} & =\frac{8 \pi G^{2} \alpha_{0}^{2}}{5} \frac{\left[\left|\tilde{\tau}_{i i}\right|^{2}+\frac{1}{3} \tilde{\tau}_{i j}^{*} \tilde{\tau}^{i j}\right]}{\xi_{a}^{*}(\vec{x}, f) \xi^{a}(\vec{x}, f)}, \\
\sigma_{s}^{t o t} & =\frac{2 G \alpha_{a}^{0}}{f} \frac{\Im\left[\xi^{a}(\vec{x}, f) e_{i j}^{s} \tilde{\tau}^{i j *}\right]}{\xi_{a}^{*}(\vec{x}, f) \xi^{a}(\vec{x}, f)} .
\end{aligned}
$$

where $\xi^{a}(\vec{x}, f)$ is the Fourier transform of the impinging scalar GW. We now have to decompose the scalar GW polarization tensor $e_{i j}^{s}$ in a quadrupole and a monopole part, as they excite different modes in the detector. The way to do this is by expressing $e_{i j}^{s}$ in the basis defined by the five real symmetric tensors $\mathcal{D}_{i j}^{(\epsilon)}$, plus $\mathcal{D}_{i j}^{(00)}$, proportional to the identity tensor, because these tensors are directly related to the angular momentum of the excitation (see Appendix C1 and Ref. [19]).

Assuming again resonant scattering and noting that the resonance frequencies of the quadrupole and the monopole modes need not be equal, we have now two expressions for the variation of the stress energy tensor of the detector: the first, labelled $\tilde{\tau}_{i j}(f ; l=0)$ and valid for the sphere monopole mode, is proportional to $\mathcal{D}_{i j}^{(00)}$ and has resonance frequency $f=f_{n 0}$; the second, $\tilde{\tau}_{i j}(f ; l=2)$, is proportional' to $\sum_{\epsilon} F_{s}^{(\epsilon)} \mathcal{D}_{i j}^{(\epsilon)}$ and has resonance frequency $f=f_{n 2} \neq f_{n 0}$

$$
\begin{aligned}
& \tilde{\tau}_{i j}(f ; l=0)=\beta^{\prime}(f) \alpha_{a}^{0} \xi^{a}(f) \mathcal{D}_{i j}^{(00)}, \\
& \tilde{\tau}_{i j}(f ; l=2)=\beta^{\prime \prime}(f) \alpha_{a}^{0} \xi^{a}(f) \sum_{\epsilon} F_{s}^{(\epsilon)} \mathcal{D}_{i j}^{(\epsilon)},
\end{aligned}
$$

where $\beta^{\prime}(f) \neq \beta^{\prime \prime}(f)$ are the analogous of the function $\gamma(f)$ in $(3.10)$ and $F_{s}^{(\epsilon)} \equiv \mathcal{D}_{i j}^{(\epsilon)} e_{s}^{i j}(\hat{\Omega})$. We deduce then the total cross section of the monopole mode

$$
\sigma_{s}(f ; n, l=0)=\frac{\alpha_{0}^{2}}{1+\alpha_{0}^{2}} \frac{\tilde{G} M v^{2} H_{n}}{\pi} \frac{\Delta_{f_{n 0}}}{\left(f-f_{n 0}\right)^{2}+\Delta_{f_{n 0}}^{2} / 4},
$$

where $H_{n}$ is a constant depending on the monopolar mode under exam [23] and $\Delta_{f_{n 0}}$ is the resonance bandwidth. For the quadrupole modes $\mathcal{D}_{i j}^{(\epsilon)}$, the same calculation gives

$$
\sigma_{s}(f ; n, l=2)=\sum_{\epsilon} \sigma_{s}^{(\epsilon)}(f, \hat{\Omega})
$$

where

$$
\sigma_{s}^{(\epsilon)}(f, \hat{\Omega})=\frac{\alpha_{0}^{2}}{1+\alpha_{0}^{2}} \frac{\tilde{G} M v^{2} F_{n}}{2 \pi} \frac{\Delta_{f_{n 2}}}{\left(f-f_{n 2}\right)^{2}+\Delta_{f_{n 2}}^{2} / 4}\left(F_{s}^{(\epsilon)}\right)^{2}
$$

The pattern functions $F_{s}^{(\epsilon)}$ are listed in Table I: since an explicit computation yields

$$
\sum_{\epsilon}\left(F_{s}^{(\epsilon)}\right)^{2}=\frac{1}{3}
$$

the global response to scalar waves of the quadrupole modes is isotropic too, and total cross section (3.21) reads

$$
\sigma_{s}(f ; n, l=2)=\frac{\alpha_{0}^{2}}{1+\alpha_{0}^{2}} \frac{\tilde{G} M v^{2} F_{n}}{6 \pi} \frac{\Delta_{f_{n 2}}}{\left(f-f_{n 2}\right)^{2}+\Delta_{f_{n 2}}^{2} / 4} .
$$

As the quadrupole modes are sensitive to scalar and to tensor waves, the angular dependence of each cross section could make it possible, in principle, to guess the polarization. For instance, considering the $m=0$ mode, $F_{s}^{(0)}(\theta, \phi)$ gets a maximum for $\theta=\phi=0$, while $F_{+}^{(0)}(0,0)=F_{\times}^{(0)}(0,0)=0$.

\footnotetext{
${ }^{4}$ The $l=2$ part of $e_{i j}^{s}$ expanded in the $\mathcal{D}_{i j}^{(\epsilon)}$ basis is $2 \sum_{\epsilon} F_{s}^{(\epsilon)} \mathcal{D}_{i j}^{(\epsilon)}$.
} 
TABLE I. Angular dependence of the sphere pattern functions for the three independent polarizations of a scalar tensor GW. Notice that the pattern functions of the $\epsilon=2 c$ mode coincide with the ones of the interferometer introduced in (3.3)

\begin{tabular}{cccc}
\hline \hline Mode $(\epsilon)$ & $F_{+}^{(\epsilon)}(\theta, \phi)$ & $F_{\times}^{(\epsilon)}(\theta, \phi)$ & $F_{s}^{(\epsilon)}(\theta, \phi)$ \\
\hline $2 s$ & $-\cos \theta \cos 2 \phi$ & $-\frac{1}{2}\left(1+\cos ^{2} \theta\right) \sin 2 \phi$ & $-\frac{1}{2} \sin _{2} \sin ^{2} \theta$ \\
$2 c$ & $-\cos \theta \sin 2 \phi$ & $\frac{1}{4}(3+\cos 2 \theta) \cos 2 \phi$ & $\frac{1}{2} \cos 2 \phi \sin { }^{2} \theta$ \\
$1 s$ & $-\sin \theta \sin \phi$ & $\frac{1}{2} \sin 2 \theta \cos \phi$ & $-\frac{1}{2} \cos \phi \sin 2 \theta$ \\
$1 c$ & $-\sin \theta \cos \phi$ & $-\frac{1}{2} \sin 2 \theta \sin \phi$ & $\frac{1}{2} \sin \phi \sin 2 \theta$ \\
0 & 0 & $\frac{\sqrt{3}}{2} \sin ^{2} \theta$ & $\frac{\sqrt{3}}{6}\left(3 \cos ^{2} \theta-1\right)$ \\
\hline \hline
\end{tabular}

The integration of (3.20) and (3.24) gives, respectively

$$
\Sigma_{s}(n ; l=0)=\frac{2 \alpha_{0}^{2}}{1+\alpha_{0}^{2}} \tilde{G} M v^{2} H_{n},
$$

and

$$
\Sigma_{s}(n ; l=2)=\frac{\alpha_{0}^{2}}{1+\alpha_{0}^{2}} \frac{\tilde{G} M v^{2} F_{n}}{3}
$$

3. Non minimally coupled scalar GWs: $\tilde{q}_{a} \neq 0$

In this section we present the full generalization of the result presented before to the case in which $\tilde{q}_{a}$ is small but not exactly null. We will follow step by step the procedure outlined in Ref. [23]. Further details for the general multi scalar metric theory can be found in Ref. [26].

a. The energy momentum conservation law

First let us consider the energy momentum tensor $\tilde{T}^{\mu \nu}$ of the resonant sphere and write the linearized conservation law (2.8) in momentum space. Denoting by $\tilde{\tau}^{\mu \nu}(x), \tilde{\tau}(x)$ the linear part of $\tilde{T}^{\mu \nu}, \tilde{T}$ we get

$$
\partial_{\mu} \tilde{\tau}^{\mu \nu}(x)+\tilde{\sigma}_{a}(x) \partial^{\nu} \xi^{a}(x)=0
$$

which in momentum space reads

$$
k_{\mu} \tilde{\tau}^{\mu \nu}(k)+\tilde{\sigma}_{a}(k) *\left[k^{\nu} \xi^{a}(k)\right]=0 .
$$

The reality of $\tilde{\tau}^{\mu \nu}(x)$ and $\xi^{a}(x)$ implies $\tilde{\tau}^{\mu \nu *}(k)=\tilde{\tau}^{\mu \nu}(-k)$ and $\xi^{a *}(k)=\xi^{a}(-k)$, with $k=(\vec{k}, \omega)$ and $\omega \equiv k_{0}=|\vec{k}|$. The asterisk in (3.28) stands for the four dimensional convolution product. Now we proceed to express $\tilde{\tau}_{00}(k)$ in terms of $\tilde{\tau}_{i j}(k)$.

For a particle of mass $m$, 2.10) defines the relation between the scalar charge densities and the components of the energy momentum tensors. Integration over all particles of the resonant sphere gives

$$
\tilde{\sigma}_{a}(x)=-\tilde{q}_{a} \tilde{\tau}(x)
$$

and therefore the four equations (3.28) in momentum space read (with $\left.\tilde{\tau}_{\mu \nu}(k) \equiv \tilde{\tau}_{\mu \nu}\right)$

$$
\begin{gathered}
k_{0} \tilde{\tau}^{00}+k_{i} \tilde{\tau}^{0 i}-\tilde{q}_{a} \tilde{\tau} *\left[k^{0} \xi^{a}(k)\right]=0, \\
k_{0} \tilde{\tau}^{0 i}+k_{j} \tilde{\tau}^{i j}-\tilde{q}_{a} \tilde{\tau} *\left[k^{i} \xi^{a}(k)\right]=0 .
\end{gathered}
$$

The wave travels along the direction $\hat{\Omega}$, and so $k_{i}=k_{0} \hat{\Omega}_{i}$ because the scalar fields are massless. Subtracting the contraction of (3.31) with $\hat{\Omega}_{i}$ from $(3.30)$ gives then

$$
\tilde{\tau}_{00}=\tilde{\tau}_{i j} \hat{\Omega}^{i} \hat{\Omega}^{j}
$$

a relation which holds in minimally coupled scalar tensor theories too [26].

We now compute again, in the case $\tilde{q}_{a} \neq 0$, the quantities entering the cross sections: the incoming energy flux, the power emitted by the detector in GWs and the interference power (see Ref. [14]). The calculation strictly follows that of Ref. [23] for Brans-Dicke theory which has been generalized in Ref. [26] to multi scalar metric theory. These latter results are recovered in the limit $\tilde{q}_{a} \rightarrow 0$. 


\section{b. The incoming energy flux}

Let us start with the incoming energy flux which is independent from the direction of the incoming GW thanks to the symmetry of the detector. We will simplify here the calculations assuming the incoming direction to be coincident with the $\hat{z}$ axis of the detector frame. Later we will recover the general expression for an arbitrary direction.

The incoming flux is computed given the energy momentum pseudotensor of the gravitational field. At second order in the linear expansion, defined in (2.14)

$$
\begin{aligned}
\tilde{t}_{\mu \nu}^{(2)}= & \frac{2}{8 \pi G}\left\{-\left(\partial_{a} \alpha_{b}\right)_{0} \xi^{a} \partial_{\mu} \partial_{\nu} \xi^{b}+\frac{1}{2} \alpha_{b}^{0} \eta^{\rho \sigma}\left(\partial_{\mu} \tilde{h}_{\nu \rho}+\partial_{\nu} \tilde{h}_{\mu \rho}-\partial_{\rho} \tilde{h}_{\mu \nu}\right) \partial_{\sigma} \xi^{b}+\right. \\
& -\left(\partial_{a} \alpha_{b}\right)_{0} \eta_{\mu \nu} \eta^{\rho \sigma} \xi^{a} \partial_{\rho} \partial_{\sigma} \xi^{b}+\frac{1}{2} \alpha_{b}^{0} \eta_{\mu \nu} \eta^{\epsilon \gamma} \eta^{\rho \sigma}\left(\partial_{\epsilon} \tilde{h}_{\gamma \rho}+\partial_{\gamma} \tilde{h}_{\epsilon \rho}-\partial_{\rho} \tilde{h}_{\gamma \epsilon}\right) \partial_{\sigma} \xi^{b}+ \\
& \left.-\left[\left(\partial_{a} \alpha_{b}\right)_{0}+\alpha_{a}^{0} \alpha_{b}^{0}-\gamma_{a b}^{0}\right] \partial_{\mu} \xi^{a} \partial_{\nu} \xi^{b}-\left[\left(\partial_{a} \alpha_{b}\right)_{0}-\frac{1}{2} \alpha_{a}^{0} \alpha_{b}^{0}-\frac{1}{2} \gamma_{a b}^{0}\right] \eta_{\mu \nu} \eta^{\rho \sigma} \partial_{\rho} \xi^{a} \partial_{\sigma} \xi^{b}\right\}+ \\
& -\frac{1}{8 \pi G}\left(\tilde{R}_{\mu \nu}^{(2)}-\frac{1}{2} \eta_{\mu \nu} \eta^{\alpha \beta} \tilde{R}_{\alpha \beta}^{(2)}+\frac{1}{2} \eta_{\mu \nu} \tilde{h}^{\alpha \beta} \tilde{R}_{\alpha \beta}^{(1)}-\frac{1}{2} \tilde{h}_{\mu \nu} \eta^{\alpha \beta} \tilde{R}_{\alpha \beta}^{(1)}\right)
\end{aligned}
$$

where

$$
\begin{aligned}
\tilde{R}_{\mu \nu}^{(2)}= & \frac{1}{2} \tilde{h}^{\alpha \rho}\left(\partial_{\mu} \partial_{\nu} \tilde{h}_{\alpha \rho}-\partial_{\mu} \partial_{\rho} \tilde{h}_{\alpha \nu}-\partial_{\mu} \partial_{\alpha} \tilde{h}_{\nu \rho}+\partial_{\alpha} \partial_{\rho} \tilde{h}_{\mu \nu}\right)+ \\
& +\frac{1}{4}\left(\partial_{\mu} \tilde{h}_{\alpha \rho}+\partial_{\alpha} \tilde{h}_{\mu \rho}-\partial_{\rho} \tilde{h}_{\alpha \mu}\right)\left(\partial_{\alpha} \tilde{h}_{\nu}^{\rho}+\partial_{\nu} \tilde{h}^{\alpha \rho}-\partial_{\rho} \tilde{h}_{\nu}^{\alpha}\right)+ \\
& -\frac{1}{4}\left(\partial_{\mu} \tilde{h}_{\nu \rho}+\partial_{\nu} \tilde{h}_{\mu \rho}-\partial_{\rho} \tilde{h}_{\mu \nu}\right)\left(2 \partial_{\alpha} \tilde{h}^{\alpha \rho}-\partial^{\rho} \tilde{h}\right)
\end{aligned}
$$

is the Ricci tensor linearized to second order in the fields. We keep in mind that [26]

$$
\tilde{h}_{\mu \nu}=\left(\begin{array}{cccc}
0 & 0 & 0 & 0 \\
0 & \mathcal{E}^{+}+2 \alpha_{a}^{0} \xi^{a} & \mathcal{E}^{\times} & 0 \\
0 & \mathcal{E}^{\times} & -\mathcal{E}^{+}+2 \alpha_{a}^{0} \xi^{a} & 0 \\
0 & 0 & 0 & 0
\end{array}\right),
$$

and denote by $\langle\ldots\rangle$ the integration over a 3 dimensional space region with linear dimensions much bigger than the GWs wavelength. Substituting (3.35) into (3.33) we obtain the total scalar tensor energy flux coming from the $\hat{z}$ direction

$$
\Phi(f)=\Phi_{h}+\Phi_{s}=\hat{z}<\tilde{t}_{0 z}^{(2)}>=\frac{\pi f^{2}}{2 G}\left\{\left|\mathcal{E}_{+}\right|^{2}+\left|\mathcal{E}_{\times}\right|^{2}+4 \gamma_{a b}^{0} \xi^{a *}(\vec{x}, f) \xi^{b}(\vec{x}, f)\right\} .
$$

c. The scattering amplitude and the energy cross sections

Let us consider a GW impinging onto our spherical resonant detector. At large distances, $R=|\vec{x}|$, from the detector

$$
\xi^{a}(\vec{x}, t) \rightarrow\left[\xi^{a}(\vec{x}, f) e^{i \vec{k} \cdot \vec{x}}+\Delta^{a}(\vec{x}, f) \frac{e^{2 \pi i f R}}{R}\right] e^{-2 \pi i f t},
$$

where $\Delta^{a}(\vec{x}, f)$ is the scattering amplitude relative to the ath scalar field. It obeys the usual reality condition $\Delta^{a}(\vec{x}, f)=\Delta^{a *}(\vec{x},-f)$. Using the scalar field equation (2.4), under the hypothesis that the quadrupole approximation holds, the scattering amplitude can be written in terms of $\tilde{\tau}^{\mu \nu}(x)$ as

$$
\begin{aligned}
\Delta^{a}(\vec{x}, \omega) & \simeq G \int \mathrm{d}^{3} x^{\prime}\left(\alpha_{0}^{a}-\tilde{q}^{a}\right) \tilde{\tau}\left(\vec{x}^{\prime}, \omega\right) e^{-i \vec{k} \cdot \vec{x}^{\prime}} \\
& =G\left(\alpha_{0}^{a}-\tilde{q}^{a}\right) \tilde{\tau}_{i j}(k)\left(\delta^{i j}-\hat{\Omega}^{i} \hat{\Omega}^{j}\right),
\end{aligned}
$$

where we have expressed $\tilde{\tau}_{00}(k)$ in terms of the space like components of the Fourier transform of the energy momentum tensor by making use of (3.32).

Let us turn then to the detailed calculation of the energy cross section, referring ourselves again to Ref. 23]. From (3.38) we find the scattering power to be 


$$
P^{s c a t}=\frac{2 \pi f^{2}}{G} \int \mathrm{d} \hat{\Omega} \Delta_{a}(\vec{x}, f) \Delta^{a *}(\vec{x}, f)=\frac{16 \pi^{2} G f^{2}}{5}\left\{\left|\tilde{\tau}_{i i}\right|^{2}+\frac{1}{3} \tilde{\tau}_{i j}^{*} \tilde{\tau}^{i j}\right\}\left(\alpha_{0}^{2}-2 \tilde{q}_{a} \alpha_{0}^{a}+\tilde{q}^{2}\right),
$$

where $\tilde{q}^{2}=\tilde{q}_{a} \tilde{q}^{a}$. Furthermore, the interference between the incident plane wave and the scattered wave gives

$$
P^{i n t}=\frac{2 f}{G} \Im\left[\int \mathrm{d} \hat{\Omega} \xi_{a}(\vec{x}, f) \Delta^{a *}(\vec{x}, f) \delta(1-\hat{k} \cdot \hat{x})\right]=4 \pi f \Im\left\{\xi_{a}^{*}(\vec{x}, f)\left(\alpha_{0}^{a}-\tilde{q}^{a}\right) \tilde{\tau}_{i j} e_{s}^{i j}\right\} .
$$

The scattering and total cross sections are then

$$
\begin{aligned}
\sigma_{s}^{s c a t} & =\frac{P^{s c a t}}{\Phi_{s}}=\frac{8 \pi G^{2}}{5}\left(\alpha_{0}^{2}-2 \tilde{q}_{a} \alpha_{0}^{a}+\tilde{q}^{2}\right) \frac{\left\{\left|\tilde{\tau}_{i i}\right|^{2}+\frac{1}{3} \tilde{\tau}_{i j}^{*} \tilde{\tau}^{i j}\right\}}{\xi_{c}^{*} \xi^{c}} \\
\sigma_{s}^{t o t} & =-\frac{P^{i n t}}{\Phi_{s}}=\frac{2 G}{f} \frac{\Im\left\{\left(\alpha_{a}^{0}-\tilde{q}_{a}\right) \xi^{a *} \tilde{\tau}_{i j} e_{s}^{i j}\right\}}{\xi_{c}^{*} \xi^{c}}
\end{aligned}
$$

where we have put $\xi^{c} \equiv \xi^{c}(\vec{x}, f)$. Expanding now in the $\mathcal{D}_{i j}^{(00)}, \mathcal{D}_{i j}^{(\epsilon)}$ basis, we can decompose $\tilde{\tau}_{i j}$ into an $l=0$ part and an $l=2$ part

$$
\begin{aligned}
& \tilde{\tau}_{i j}(f ; l=0)=\zeta^{\prime}(f)\left(\alpha_{a}^{0}-\tilde{q}_{a}\right) \xi^{a} \mathcal{D}_{i j}^{(00)}, \quad f=f_{n 0}, \\
& \tilde{\tau}_{i j}(f ; l=2)=\zeta^{\prime \prime}(f)\left(\alpha_{a}^{0}-\tilde{q}_{a}\right) \xi^{a} \sum_{\epsilon} F_{s}^{(\epsilon)}(\hat{\Omega}) \mathcal{D}_{i j}^{(\epsilon)}, \quad f=f_{n 2},
\end{aligned}
$$

with $\zeta^{\prime}(f) \neq \zeta^{\prime \prime}(f)$ defined as $\beta^{\prime}(f)$ and $\beta^{\prime \prime}(f)$ in (3.18) and (3.19). Hence, the monopole and the quadrupole total cross sections become

$$
\begin{aligned}
& \sigma_{s}(f ; n, l=0) \equiv \frac{2 G}{f} \Im\left(\zeta^{\prime}\right) \frac{L\left(\xi^{a} ; \alpha_{a}^{0}, \tilde{q}_{a}\right)}{\xi_{c}^{*} \xi^{c}} \\
& \sigma_{s}(f ; n, l=2) \equiv \frac{2 G}{f} \Im\left(\zeta^{\prime \prime}\right) \sum_{\epsilon}\left(F_{s}^{(\epsilon)}\right)^{2} \frac{L\left(\xi^{a} ; \alpha_{a}^{0}, \tilde{q}_{a}\right)}{\xi_{c}^{*} \xi^{c}}
\end{aligned}
$$

where

$$
L\left(\xi^{a} ; \alpha_{a}^{0}, \tilde{q}_{a}\right) \equiv\left|\alpha_{a}^{0} \xi^{a}\right|^{2}+\left|\tilde{q}_{a} \xi^{a}\right|^{2}-\left(\alpha_{a}^{0} \tilde{q}_{b} \xi^{a *} \xi^{b}+\tilde{q}_{a} \alpha_{b}^{0} \xi^{a *} \xi^{b}\right) .
$$

By using (3.41) and (3.43)-(3.46) with the analogous of (3.9) with $\sigma_{s}$ replacing $\sigma_{h}$, and assuming once again resonant scattering, we get the final form for the monopole and quadrupole total cross sections

$$
\begin{aligned}
& \sigma_{s}(f ; n, l=0) \equiv \frac{\eta_{0}}{\pi f^{2}\left(\alpha_{0}^{2}-2 \tilde{q}_{a} \alpha_{0}^{a}+\tilde{q}^{2}\right)} \frac{\Delta_{f_{n 0}}^{2} / 4}{\left(f-f_{n 0}\right)^{2}+\Delta_{f_{n 0}}^{2} / 4} \frac{L\left(\xi^{a} ; \alpha_{a}^{0}, \tilde{q}_{a}\right)}{\xi_{c}^{*} \xi^{c}}, \\
& \sigma_{s}(f ; n, l=2) \equiv \frac{15 \eta_{2}}{\pi f^{2}\left(\alpha_{0}^{2}-2 \tilde{q}_{a} \alpha_{0}^{a}+\tilde{q}^{2}\right)} \frac{\Delta_{f_{n 2}}^{2} / 4}{\left(f-f_{n 2}\right)^{2}+\Delta_{f_{n 2}}^{2} / 4} \sum_{\epsilon}\left(F_{s}^{(\epsilon)}\right)^{2} \frac{L\left(\xi^{a} ; \alpha_{a}^{0}, \tilde{q}_{a}\right)}{\xi_{c}^{*} \xi^{c}} .
\end{aligned}
$$

We still have to evaluate $\eta_{0}$ and $\eta_{2}$. This is done remembering their definition as the ratio between the power $P^{s c a t} \equiv P^{(n ; l)}$ reemitted as gravitational waves by the vibrations of the sphere and the oscillatory energy $E_{o s c}^{(n ; l)}$ dissipated by the sphere itself

$$
\eta_{0}=\frac{P^{(n ; 0)}}{2 \pi \Delta_{f_{n 0}} E_{o s c}^{(n ; 0)}}, \quad \eta_{2}=\frac{P^{(n ; 2)}}{2 \pi \Delta_{f_{n 2}} E_{o s c}^{(n ; 2)}} .
$$

The oscillatory energy is that evaluated in Refs. [20,23], since it doesn't depend on $\tilde{q}_{a}$. The calculation of the reemitted power follows that of Ref. 23,26]. The only difference consists in replacing $\alpha_{a}^{0}$ with $\alpha_{a}^{0}-\tilde{q}_{a}$. Therefore, omitting the uninteresting details of the calculation, we get 


$$
\begin{aligned}
& \eta_{0}=4 G \frac{M v^{2} f_{n 0}^{2} H_{n}}{\Delta_{f_{n 0}}}\left\{\alpha_{0}^{2}-2 \tilde{q}_{a} \alpha_{0}^{a}+\tilde{q}^{2}\right\}, \\
& \eta_{2}=\frac{2 G}{15} \frac{M v^{2} f_{n 2}^{2} F_{n}}{\Delta_{f_{n 2}}}\left\{\alpha_{0}^{2}-2 \tilde{q}_{a} \alpha_{0}^{a}+\tilde{q}^{2}\right\} .
\end{aligned}
$$

Finally the cross sections assume the following simple forms

$$
\begin{aligned}
& \sigma_{s}(f ; n, l=0) \equiv \frac{G M v^{2} H_{n}}{\pi} \frac{\Delta_{f_{n 0}}}{\left(f-f_{n 0}\right)^{2}+\Delta_{f_{n 0}}^{2} / 4} \frac{L\left(\xi^{a} ; \alpha_{a}^{0}, \tilde{q}_{a}\right)}{\xi_{c}^{*} \xi^{c}} \\
& \sigma_{s}(f ; n, l=2) \equiv \frac{G M v^{2} F_{n}}{2 \pi} \frac{\Delta_{f_{n 2}}}{\left(f-f_{n 2}\right)^{2}+\Delta_{f_{n 2}}^{2} / 4} \sum_{\epsilon}\left(F_{s}^{(\epsilon)}\right)^{2} \frac{L\left(\xi^{a} ; \alpha_{a}^{0}, \tilde{q}_{a}\right)}{\xi_{c}^{*} \xi^{c}}
\end{aligned}
$$

These expressions can be made more manageable by expanding $L\left(\xi^{a} ; \alpha_{a}^{0}, \tilde{q}_{a}\right)$ in powers of $\tilde{q}_{a} \ll \alpha_{a}^{0} \ll 1$, an ordering relation which follows from the weak field limit of (2.7).

First, an analogous calculation to that for $\Delta^{a}(\vec{x}, f)$ gives 26$]$

$$
\xi^{a}(\vec{x}, f)=\frac{G}{R}\left(\alpha_{0}^{a}-\tilde{q}^{a}\right) \tilde{\tau}_{i j}^{\prime} e_{s}^{i j}
$$

where now $\tau_{i j}^{\prime}=\tilde{\tau}_{i j}^{\prime}(k)$ is the Fourier transform of the space components of the stress energy tensor of the source located at a great distance $R$ from the antenna; $\tilde{\tau}_{i j}^{\prime}$ is related to the Fourier transform of the variation of the quadrupole moment $Q_{i j}^{\prime}(f)$ of the source by 14 .

$$
Q_{i j}^{\prime}(f)=-\frac{1}{2 \pi^{2} f^{2}} \tilde{\tau}_{i j}^{\prime}
$$

Therefore, taking into account (3.56), an explicit evaluation gives

$$
L\left(\xi^{a} ; \alpha_{a}^{0}, \tilde{q}_{a}\right)=\frac{4 \pi^{4} f^{4} G^{2}}{R^{2}}\left|Q_{i j}^{\prime} e_{s}^{i j}\right|^{2} \alpha_{0}^{4}\left\{1-4 \frac{\tilde{q}_{a} \alpha_{0}^{a}}{\alpha_{0}^{2}}+2 \frac{\tilde{q}^{2}}{\alpha_{0}^{2}}+4 \frac{\left(\tilde{q}_{a} \alpha_{0}^{a}\right)^{2}}{\alpha_{0}^{4}}-4 \frac{\tilde{q}_{a} \alpha_{0}^{a} \tilde{q}^{2}}{\alpha_{0}^{4}}+\frac{\tilde{q}^{4}}{\alpha_{0}^{4}}\right\},
$$

and

$$
\xi_{c}^{*} \xi^{c}=\frac{4 \pi^{4} f^{4} G^{2}}{R^{2}}\left|Q_{i j}^{\prime} e_{s}^{i j}\right|^{2} \alpha_{0}^{2}\left\{1-2 \frac{\tilde{q}_{a} \alpha_{0}^{a}}{\alpha_{0}^{2}}+\frac{\tilde{q}^{2}}{\alpha_{0}^{2}}\right\} .
$$

Expanding this ratio in powers of $\tilde{q}_{a}$ yields

$$
\frac{L\left(\xi^{a} ; \alpha_{a}^{0}, \tilde{q}_{a}\right)}{\xi_{a}^{*} \xi^{a}} \simeq \alpha_{0}^{2}-2 \tilde{q}_{a} \alpha_{0}^{a}+\tilde{q}^{2}+\ldots
$$

We can finally compute the $\tilde{q}_{a}$ dependent terms in the cross sections.

The monopole cross section at the lowest order in $\tilde{q}_{a}$ reads

$$
\sigma_{s}(f ; n, l=0) \simeq \frac{1}{1+\alpha_{0}^{2}} \frac{\tilde{G} M v^{2} H_{n}}{\pi} \frac{\Delta_{f_{n 0}}}{\left(f-f_{n 0}\right)^{2}+\Delta_{f_{n 0}}^{2} / 4}\left\{\alpha_{0}^{2}-2 \tilde{q}_{a} \alpha_{0}^{a}\right\}
$$

where we have reintroduced the effective Newton's gravitational constant $\tilde{G}$.

Analogously, the quadrupole cross section for any mode $\epsilon$ writes, at first order

$$
\sigma_{s}^{(\epsilon)}(f, \hat{\Omega}) \simeq \frac{1}{1+\alpha_{0}^{2}} \frac{\tilde{G} M v^{2} F_{n}}{2 \pi} \frac{\Delta_{f_{n 2}}}{\left(f-f_{n 2}\right)^{2}+\Delta_{f_{n 2}}^{2} / 4}\left\{\alpha_{0}^{2}-2 \tilde{q}_{a} \alpha_{0}^{a}\right\}\left(F_{s}^{(\epsilon)}\right)^{2} .
$$

Summing over $\epsilon,(\sqrt{3.23})$ gives

$$
\sigma_{s}(f ; n, l=2) \simeq \frac{1}{1+\alpha_{0}^{2}} \frac{\tilde{G} M v^{2} F_{n}}{6 \pi} \frac{\Delta_{f_{n 2}}}{\left(f-f_{n 2}\right)^{2}+\Delta_{f_{n 2}}^{2} / 4}\left\{\alpha_{0}^{2}-2 \tilde{q}_{a} \alpha_{0}^{a}\right\} .
$$




\section{DETECTION OF A STOCHASTIC GW BACKGROUND}

Our aim is to generalize the standard analysis about the detectability of the spin 2 stochastic GW background 28 30 to the case of the general scalar tensor theory outlined in Sec. IIB. Within this framework we introduce a density of scalar gravitational radiation $\rho_{s}$ in addition to the standard tensor one $\rho_{h}$. If we assume, as in the tensor case, that the scalar background is isotropic, unpolarized, stationary and Gaussian, it is completely described in terms of the (dimensionless) spectrum

$$
\Omega_{s}=\frac{1}{\rho_{c}} \frac{\mathrm{d} \rho_{s}}{\mathrm{~d} \ln f}
$$

where $\mathrm{d} \rho_{s}$ is the energy density of the scalar gravitational radiation in the frequency range $f$ to $f+\mathrm{d} f$ and $\rho_{c}$ is the critical density required (today) to close the universe

$$
\rho_{c}=\frac{3 H_{0}^{2}}{8 \pi \tilde{G}} .
$$

$H_{0}$ is the present value of the Hubble constant. Notice that, although we study a scalar tensor theory we normalize the scalar gravitational spectrum to the value of $\rho_{c}$ recovered in general relativity. This choice has been taken to have a direct comparison between the tensor only and the scalar tensor framework. The present value of the Hubble expansion rate is usually written as $H_{0}=h_{0} \times 100 \mathrm{~km} \mathrm{~s}^{-1} \mathrm{MPc}^{-1}$, where $h_{0}(=0.6 \div 0.7)$ is a dimensionless factor that parametrizes the experimental uncertainty affecting the value of $H_{0}$. As a consequence of this definition the quantity $h_{0}^{2} \Omega_{s}(f)$ is independent of $h_{0}$, and, thus, more suitable to characterize the stochastic GW background.

\section{A. The signal to noise ratio for scalar tensor GW stochastic background}

From the experimental side, the signal induced in the detector output by a stochastic GW background is indistinguishable from the intrinsic noise of the detector itself. Unless the amplitude of the signal is very large, then, the subtraction of an a priori estimate of the detector noise cannot be confidently applied to the data. This implies that in order to detect a stochastic GW background, we should rather analyze the correlated fluctuations of the outputs of, at least, two detectors with no common sources of noise (a condition usually verified for widely separated detector sites). The cross correlation among detectors is advantageous also from the point of view of the minimum detectable signal. It can be shown 29,31] that, under the same experimental conditions, the minimum detectable signal in the correlation of two detectors can be even three orders of magnitude smaller than the one detectable with a single detector.

The problem of the optimal processing of the the detector outputs for the detection of the stochastic GW background (tensor and scalar) has been considered by various authors [9,28 30], and extensively reviewed in Ref. [31]. This analysis can be generalized with minor modifications to the case of the general scalar tensor theory considered here.

The signal present at the output of each detector can be written as (we consider the case of two detectors)

$$
s_{k}(t)=n_{k}(t)+\tilde{h}_{k}^{e f f}(t)
$$

where we have indicated with $\tilde{h}_{k}^{e f f}$ the gravitational strain due to the stochastic GW background and with $n$ the intrinsic noise of the detector, while $k=1,2$ labels the detector to which each quantity is referred. The noise is assumed to be stationary, Gaussian and statistically independent on the gravitational strain. Furthermore, the assumption that the noises in the two detectors are uncorrelated implies that the ensemble average of their Fourier components satisfies

$$
<n_{k}^{*}(f) n_{l}\left(f^{\prime}\right)>=\delta\left(f-f^{\prime}\right) \delta_{k l} \frac{1}{2} S_{n}^{(k)}(|f|),
$$

where $S_{n}^{(k)}(|f|)$ is the (one sided) noise power spectrum for the $k$ th detector. Given an observation time $T$, the correlation "signal" is defined as follows

$$
S=\int_{-T / 2}^{T / 2} \mathrm{~d} t \int_{-T / 2}^{T / 2} \mathrm{~d} t^{\prime} s_{1}(t) s_{2}\left(t^{\prime}\right) Q\left(t-t^{\prime}\right)
$$


where $Q$ is a real filter function that, for any form of the signal, is chosen in order to maximize the signal to noise ratio (SNR) associated to $S$ [30].

The statistical treatment of the signal $S$ defined in (4.5) starts with the plane wave expansion of the metric perturbations. The effective GW exciting the detector at position $\vec{x}$ writes

$$
\tilde{h}_{i j}^{e f f}(t, \vec{x})=\int_{-\infty}^{\infty} \mathrm{d} f \int_{S^{2}} \mathrm{~d} \hat{\Omega}\left[h_{A}(f, \hat{\Omega}) e_{i j}^{A}(\hat{\Omega})+2\left(\alpha_{a}^{0}-\tilde{q}_{a}\right) \xi^{a}(f, \hat{\Omega}) e_{i j}^{s}(\hat{\Omega})\right] e^{2 \pi i f(t-\hat{\Omega} \cdot \vec{x})},
$$

where, as a consequence of the reality of $\tilde{h}_{i j}^{e f f}(t, \vec{x})$, we have that $h_{A}^{*}(f)=h_{A}(-f)$ and $\xi^{a *}(f)=\xi^{a}(-f) ; \hat{\Omega}$ is the unit vector specifying the direction of the incoming GW.

In terms of the detector tensor, the GW strain sensed by the detector $k$ located at $\vec{x}_{k}$ is given by

$$
\tilde{h}_{k}^{e f f}(t) \equiv \tilde{h}^{e f f}\left(t, \vec{x}_{k}\right)=\tilde{h}_{i j}^{e f f}\left(t, \vec{x}_{k}\right) \mathcal{D}_{k}^{i j},
$$

and (4.7), keeping into account (4.6) and the definitions (3.3), it can be rewritten as

$$
\tilde{h}_{k}^{e f f}(t)=\int_{-\infty}^{\infty} \mathrm{d} f \int_{S^{2}} \mathrm{~d} \hat{\Omega}\left[h_{A}(f, \hat{\Omega}) F_{k}^{A}(\hat{\Omega})+2\left(\alpha_{a}^{0}-\tilde{q}_{a}^{(k)}\right) \xi^{a}(f, \hat{\Omega}) F_{k}^{s}(\hat{\Omega})\right] e^{2 \pi i f\left(t-\hat{\Omega} \cdot \vec{x}_{k}\right)} .
$$

In the following, we focus on the spin 0 contribution to the strain, i.e. the part of (4.8) depending on $\xi^{a}(f, \hat{\Omega})$ (the spin 2 contribution has been extensively treated in Ref. [28 30]). Since the scalar background is assumed stationary, Gaussian, isotropic and unpolarized in the space of the scalar fields, it can be shown (see Appendix A for further details) that the correlation functions between the Fourier amplitude $\xi^{a}(f, \hat{\Omega})$ of the waves are

$$
<\xi^{a *}(f, \hat{\Omega}) \xi^{b}\left(f^{\prime}, \hat{\Omega}\right)>=\frac{1}{1+\alpha_{0}^{2}} \gamma_{0}^{a b} \frac{3 H_{0}^{2}}{64 \pi^{3}} \frac{1}{f^{3}} \Omega_{\xi}(f) \delta\left(f-f^{\prime}\right) \delta\left(\hat{\Omega}-\hat{\Omega}^{\prime}\right),
$$

where $\Omega_{\xi}(f)$ is the spectrum of a single scalar field. As a consequence of our assumptions on the scalar background, the whole spectrum is then $\Omega_{s}=n \Omega_{\xi}$. Following the same line of reasoning applied in the case of the spin 2 waves, under the further assumptions that the detector noises are much larger in amplitude than the gravitational strain and statistically independent on the strain itself, for the SNR we obtain

$$
\mathrm{SNR}_{\xi}=\frac{\alpha_{0}^{2}-\left(\tilde{q}_{a}^{(1)}+\tilde{q}_{a}^{(2)}\right) \alpha_{0}^{a}+\tilde{q}_{a}^{(1)} \tilde{q}_{b}^{(2)} \gamma^{a b}}{1+\alpha_{0}^{2}} \frac{3 H_{0}^{2}}{8 \pi^{3}}\left[2 T \int_{0}^{\infty} \mathrm{d} f \frac{\Omega_{\xi}^{2}(f) \Gamma_{\xi}^{2}(f)}{f^{6} S_{n}^{(1)}(f) S_{n}^{(2)}(f)}\right]^{1 / 2} .
$$

The function $\Gamma_{\xi}(f)$ is the generalization to scalar fields of the usual overlap reduction function introduced in [29, 30]. This is a dimensionless function describing the reduction in sensitivity due to the different location and orientation of the two detectors, and it is given by

$$
\Gamma_{\xi}(f)=\int_{S^{2}} \mathrm{~d} \hat{\Omega} F_{1}^{s}(\hat{\Omega}) F_{2}^{s}(\hat{\Omega}) e^{2 \pi i f d \hat{\Omega} \cdot \hat{s}}
$$

where $\hat{s}$ is the unit vector along the direction connecting the two detectors and $d$ is their distance. Notice that $\Gamma_{\xi}(f)$ coincides with the scalar overlap reduction function introduced in Ref. [9] in the context of the single scalar metric theory of Brans-Dicke. The SNR obtained in Ref. [9] is also recovered specializing (4.10) by setting $\tilde{q}_{a}^{(k)}=0$ and $\alpha_{0}^{2}=\left(2 \omega_{B D}+3\right)^{-1}$.

In the following, we will consider two detectors with the same $\tilde{q}_{a}^{(1)}=\tilde{q}_{a}^{(2)}=\tilde{q}_{a}$. If we keep only linear terms in $\tilde{q}_{a}$, (4.10) becomes

$$
\mathrm{SNR}_{\xi}=\frac{\alpha_{0}^{2}-2 \tilde{q}_{a} \alpha_{0}^{a}}{1+\alpha_{0}^{2}} \frac{3 H_{0}^{2}}{8 \pi^{3}}\left[2 T \int_{0}^{\infty} \mathrm{d} f \frac{\Omega_{\xi}^{2}(f) \Gamma_{\xi}^{2}(f)}{f^{6} S_{n}^{(1)}(f) S_{n}^{(2)}(f)}\right]^{1 / 2}
$$

\footnotetext{
${ }^{5}$ This function depends only on $t-t^{\prime}$ as consequence of the assumed stationarity of both the gravitational strain and the detector noise.
} 
As pointed out before, the spin 2 contribution to the strain (4.8) is substantially the one already obtained in the literature. However the presence of the scalar fields slightly modifies the usual formula for the $\mathrm{SNR}_{h}$ of Ref. [30] introducing an overall $\alpha_{0}^{2}$ dependent factor which is absent in General Relativity

$$
\mathrm{SNR}_{h}=\frac{1}{1+\alpha_{0}^{2}} \frac{3 H_{0}^{2}}{8 \pi^{3}}\left[2 T \int_{0}^{\infty} \mathrm{d} f \frac{\Omega_{h}^{2}(f) \Gamma_{h}^{2}(f)}{f^{6} S_{n}^{(1)}(f) S_{n}^{(2)}(f)}\right]^{1 / 2} .
$$

Here $\Omega_{h}(f)$ is the usual spin 2 spectrum and

$$
\Gamma_{h}(f)=\int_{S^{2}} \mathrm{~d} \hat{\Omega} \sum_{A} F_{1}^{A}(\hat{\Omega}) F_{2}^{A}(\hat{\Omega}) e^{2 \pi i f d \hat{\Omega} \cdot \hat{s}},
$$

is the (non normalized) overlap reduction function for spin 2 waves.

The most general expression for (4.11) and (4.14) can be shown to be 30]

$$
\begin{aligned}
\Gamma_{w}(\tau)=\pi & \left\{A_{w}(\tau) \operatorname{Tr}\left(\mathcal{D}_{1}\right) \operatorname{Tr}\left(\mathcal{D}_{2}\right)+2 B_{w}(\tau) \operatorname{Tr}\left(\mathcal{D}_{1} \mathcal{D}_{2}\right)\right. \\
& +C_{w}(\tau)\left[\operatorname{Tr}\left(\mathcal{D}_{1}\right) \operatorname{Tr}\left(\mathcal{S} \mathcal{D}_{2}\right)+\operatorname{Tr}\left(\mathcal{D}_{2}\right) \operatorname{Tr}\left(\mathcal{S} \mathcal{D}_{1}\right)\right] \\
& \left.+4 D_{w}(\tau) \operatorname{Tr}\left(\mathcal{S} \mathcal{D}_{1} \mathcal{D}_{2}\right)+E_{w}(\tau) \operatorname{Tr}\left(\mathcal{S} \mathcal{D}_{1}\right) \operatorname{Tr}\left(\mathcal{S D}_{2}\right)\right\} \quad w=h, \xi
\end{aligned}
$$

where $\tau=2 \pi f d, \mathcal{S} \equiv \hat{s} \otimes \hat{s}$, and $\mathcal{D}_{k}$ is the tensor of the $k$ th detector. Following the procedure sketched in Refs. [9.29.30], the coefficients A, B, C, D, and E can be expressed as linear superpositions of Bessel functions (see Appendix B). The traces appearing in (4.15) carry information about the geometry and the relative orientations of the detectors that are correlated.

\section{B. The noise power spectrum}

\section{d. Resonant mass detectors}

Let us consider a generic multi mode resonant mass antenna, with the modes labelled by an index $N$, as for example a resonant sphere, where $N \equiv n l$.

The noise power spectrum is a resonant curve peaked at the proper frequency $f_{N}$ of the modes. It can be characterized by its value at the peak $S_{n}\left(f_{N}\right)$ and by its half height width, which gives the bandwidth of the resonant mode.

We now generalize the results of Refs. [21,27] concerning $S_{n}\left(f_{N}\right)$ of resonant spheres to the scalar tensor theory. Denoting by $\beta_{N}$ the transducer coupling factor (the fraction of the total mode energy available at the transducer output), in the case of spin 2 GWs we have

$$
S_{n}\left(h ; f_{N}\right)=\frac{1}{1+\alpha_{0}^{2}} \frac{4 \pi \tilde{G} \hbar \beta_{N}}{\Sigma_{N}}=\frac{4 \pi \hbar \beta_{n 2}}{M v^{2} F_{n}}
$$

where the expression (3.15) for $\Sigma_{N} \equiv \Sigma_{h}(n ; l=2)$ has been used.

For spin $0 \mathrm{GWs}$, since we have considered only the first order terms in the expansion in powers of $\tilde{q}_{a}$ of the integrated cross section, we write

$$
S_{n}\left(\xi ; f_{N}\right)=\frac{\alpha_{0}^{2}}{1+\alpha_{0}^{2}}\left(1-2 \frac{\tilde{q}_{a} \alpha_{0}^{a}}{\alpha_{0}^{2}}\right) \frac{4 \pi \tilde{G} \hbar \beta_{N}}{\Sigma_{N}}
$$

where $\Sigma_{N}$ is now the obvious generalization of (3.25), (3.26) to the case $\tilde{q}_{a} \neq 0$. Making explicit (4.17) for both the monopole and the quadrupole modes, we get

$$
\begin{gathered}
S_{n}\left(\xi ; f_{n 0}\right)=\frac{2 \pi \hbar \beta_{n 0}}{M v^{2} H_{n}}, \\
S_{n}\left(\xi ; f_{n 2}\right)=\frac{12 \pi \hbar \beta_{n 2}}{M v^{2} F_{n}} .
\end{gathered}
$$

Finally, let us remark that formulae like (4.16), (4.17) hold in general for any kind of detector, because the dependence of the cross section on the coupling constants $\tilde{G}, \alpha_{a}^{0}$ and $\tilde{q}_{a}$ doesn't change according to the geometrical features 
of the antenna itself. Actually, if no scalar fields are present, $\alpha_{a}^{0}=\tilde{q}_{a}=0$, 4.17) vanishes and (4.16) becomes the well known formula (4.5) of Ref. 21] for the maximum sensitivity to spin 2 waves.

The bandwidth of the resonant mode is given by

$$
\Delta_{f_{N}}=\frac{f_{N}}{Q_{N}} \Gamma_{N}^{-1 / 2}
$$

where $Q_{N}$ is the quality factor of the mode, which is of the order of $10^{7}$ and $\Gamma_{N}$ is the ratio of the wideband noise to the narrowband noise in the $N$ th resonance mode.

e. The VIRGO interferometer

In the frequency region above $2 \mathrm{~Hz}$ the noise power spectrum of the VIRGO interferometer can be approximated by the following analytical expression 32$]^{6}$

$$
S_{n}(f)=P_{1}\left(\frac{f_{0}}{f}\right)^{5}+P_{2}\left(\frac{f_{0}}{f}\right)+P_{3}\left[1+\left(\frac{f}{f_{0}}\right)^{2}\right],
$$

with

$$
f_{0}=500 \mathrm{~Hz}, \quad \mathrm{P}_{1}=3.46 \times 10^{-50} \mathrm{~Hz}^{-1}, \quad \mathrm{P}_{2}=9 \times 10^{-46} \mathrm{~Hz}^{-1}, \quad \mathrm{P}_{3}=3.24 \times 10^{-46} \mathrm{~Hz}^{-1} .
$$

In this parametrization $P_{1}$ and $P_{2}$ give the contribution of the pendulum and its internal modes to the thermal noise, respectively. $P_{3}$ controls instead the shot noise contribution. For frequency smaller than $2 \mathrm{~Hz}$ we assume that the noise power spectrum goes to infinity.

\section{Sensitivity of a pair of resonant spheres}

We consider now the correlation between two resonant spheres. As we are interested in scalar waves, we compute the correlation between the monopole modes. Since the monopole tensors are isotropic

$$
\mathcal{D}_{i j}^{1}=\mathcal{D}_{i j}^{2}=\mathcal{D}_{i j}^{(00)}=\frac{1}{2} \delta_{i j},
$$

the overlap reduction function depends only on the frequency $f$ and the relative distance $d$. From the general formula (4.15) one finds

$$
\Gamma_{\xi}(\tau)=\pi\left[\frac{9}{4} A_{\xi}(\tau)+\frac{3}{2} B_{\xi}(\tau)+\frac{3}{2} C_{\xi}(\tau)+D_{\xi}(\tau)+\frac{1}{4} E_{\xi}(\tau)\right],
$$

which explicitly reads (see Appendix B)

$$
\Gamma_{\xi}(f)=4 \pi j_{0}(\tau) .
$$

In Fig. 1 we plot this function for $d=50 \mathrm{~km}$, which is roughly the minimum distance to decorrelate seismic and e.m. noises and for $d=400 \mathrm{~km}$, which is the distance between the sites of the resonant bars NAUTILUS, in Frascati, and AURIGA in Legnaro. For $d=50 \mathrm{~km}$ we observe that the first zero of the function is around $3 \mathrm{kHz}$, which is a frequency higher than the first resonant frequency for both the solid mass and hollow sphere. For $d=400 \mathrm{~km}$, the first zero moves back at around $400 \mathrm{~Hz}$.

Restricting ourselves to metric theories $\left(\tilde{q}_{a}=0\right)$, the SNR is

$$
\mathrm{SNR}_{\xi}=\frac{\alpha_{0}^{2}}{1+\alpha_{0}^{2}} \frac{3 H_{0}^{2}}{8 \pi^{3}} \sqrt{\frac{\pi}{2} \Delta_{f_{n 0}} T} \frac{\Omega_{\xi}\left(f_{n 0}\right) \Gamma_{\xi}\left(f_{n 0}\right)}{f_{n 0}^{3} S_{n}\left(\xi ; f_{n 0}\right)},
$$

\footnotetext{
${ }^{6}$ With respect to this reference, the value of $P_{2}$ is slightly changed as can be found in http://www.virgo.infn.it/senscurve. We quote here the most recent value. We thank the referee for pointing this out.
} 
FIG. 1. The overlap reduction function $\Gamma_{\xi}(f)$ for two resonant spheres, located at relative distance $d=50 \mathrm{~km}$ (solid line) and $d=400 \mathrm{~km}$ (dashed line).

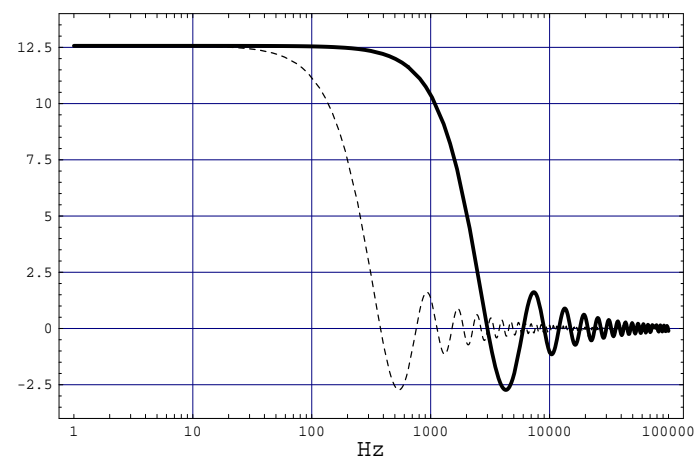

where $f_{n 0}$ is the resonance frequency for the $n$th monopole mode. To evaluate (4.25) we need the noise power spectrum at resonance $f=f_{n 0}$ given by (4.18) where we assume $\beta_{n 0}=0.1$ [21]. The only free parameter in (4.25) is $\alpha_{0}^{2}$ which can be conveniently expressed in terms of the post Newtonian Eddington parameter [10] as

$$
\frac{\alpha_{0}^{2}}{1+\alpha_{0}^{2}}=\frac{1-\gamma_{\mathrm{Edd}}}{2} .
$$

$\gamma_{\text {Edd }}$ can be measured in light deflection experiments 33 . The minimum detectable scalar spectrum, for an observation time $T=1$ year, is found imposing $\mathrm{SNR}_{\xi}=1$ in (4.25). To isolate its dependence from $\gamma_{\text {Edd }}$, in Table II we list the reduced spectrum $\Omega_{\gamma_{\mathrm{Edd}}}=\Omega_{\xi}\left(1-\gamma_{\mathrm{Edd}}\right) / 2$. We will comment in the Conclusions on the results obtained.

In Tables II, III, IV, we consider hollow and solid mass spheres made of CuAl, Al5056 and Mo, materials which have high density and high velocity of sound [21,25]. The geometrical features of such spheres are the outer diameter $\Phi$ and the ratio $\zeta$ between the inner and outer radius. We consider the first excited monopole mode, so in (4.18) we put $n=1$ and $f_{10} \equiv f_{0}$. For solid mass spheres we have $H_{1}=1.14$ 23, while for hollow spheres $H_{1}$ is a function of $\zeta$, and some interesting values are listed in the tables themselves [25].

TABLE II. Minimum detectable scalar spectrum $\left(\mathrm{SNR}_{\xi}=1, T=1\right.$ year $)$ for the correlation between the first monopole modes of two hollow spheres, with 6 meters outer diameter, made of $\mathrm{CuAl}(v=4700 \mathrm{~m} / \mathrm{s})$ at $d=50 \mathrm{~km}$.

\begin{tabular}{ccccccc}
\hline \hline$M$ (ton) & $\zeta$ & $H_{1}$ & $f_{0}(\mathrm{~Hz})$ & $\Delta_{f_{0}}(\mathrm{~Hz})$ & $\Gamma_{\xi}\left(f_{0}\right)$ & $\sqrt{S_{n}\left(\xi ; f_{0}\right)}\left(\mathrm{Hz}^{-1 / 2}\right)$ \\
\hline 832 & 0.25 & 0.73727 & 770 & 24.3 & 11.2 & $2.21 \times 10^{-24}$ \\
740 & 0.50 & 0.49429 & 609 & 19.2 & 11.7 & $2.86 \times 10^{-24}$ \\
489 & 0.75 & 0.4307 & 498 & 15.7 & 12.0 & $3.77 \times 10^{-24}$ \\
230 & 0.90 & 0.42043 & 455 & 14.4 & 12.1 & $5.57 \times 10^{-24}$ \\
\hline \hline
\end{tabular}

TABLE III. The same of Table II in the case of two 31 ton Mo $(v=5700 \mathrm{~m} / \mathrm{s})$ hollow spheres.

\begin{tabular}{ccccccc}
\hline \hline$\Phi(\mathrm{m})$ & $\zeta$ & $H_{1}$ & $f_{0}(\mathrm{~Hz})$ & $\Delta_{f_{0}}(\mathrm{~Hz})$ & $\Gamma_{\xi}\left(f_{0}\right)$ & $\sqrt{S_{n}\left(\xi ; f_{0}\right)}\left(\mathrm{Hz}^{-1 / 2}\right)$ \\
\hline 1.82 & 0.25 & 0.73727 & 3027 & 95.7 & 0.1 & $9.5 \times 10^{-24}$ \\
1.88 & 0.50 & 0.49429 & 2304 & 72.9 & 3.5 & $1.1 \times 10^{-23}$ \\
2.16 & 0.75 & 0.4307 & 1650 & 52.2 & 7.2 & $1.2 \times 10^{-23}$ \\
2.78 & 0.90 & 0.42043 & 1170 & 37.0 & 9.6 & $1.3 \times 10^{-23}$ \\
\hline \hline
\end{tabular}

Similar analysis of correlations can be repeated for the quadrupole vibrational modes of the resonant spheres, which can be excited by both spin 0 and spin 2 waves. 
TABLE IV. The same of Table II in the case of two solid spheres made of $\mathrm{CuAl}(v=4700 \mathrm{~m} / \mathrm{s})$ and $\mathrm{Al} 5056(v=5440 \mathrm{~m} / \mathrm{s})$.

\begin{tabular}{ccccccc}
\hline \hline & $M$ (ton) & $\Phi(\mathrm{m})$ & $f_{0}(\mathrm{~Hz})$ & $\Delta_{f_{0}}(\mathrm{~Hz})$ & $\Gamma_{\xi}\left(f_{0}\right)$ & $\sqrt{S_{n}\left(\xi ; f_{0}\right)}\left(\mathrm{Hz}^{-1 / 2}\right)$ \\
\hline \multirow{2}{*}{ CuAl } & 105 & 3 & 1672 & 52.9 & 7.1 & $5.0 \times 10^{-24}$ \\
& 167 & 3.5 & 1433 & 45.3 & 8.3 & $4.0 \times 10^{-24}$ \\
& 250 & 4 & 1254 & 39.7 & 9.2 & $3.2 \times 10^{-24}$ \\
\hline \multirow{2}{*}{ Al5056 } & 38 & 3 & 1935 & 61.2 & 5.6 & $7.2 \times 10^{-7}$ \\
& 60 & 3.5 & 1658 & 52.4 & 7.1 & $5.2 \times 10^{-7}$ \\
& 90 & 4 & 1451 & 45.9 & 8.3 & $4.7 \times 10^{-24}$ \\
\hline \hline
\end{tabular}

Let us face the calculation of the overlap reduction function. The quadrupole tensors $\mathcal{D}_{i j}^{(\epsilon)}$ are traceless, therefore the only non vanishing terms in the overlap reduction function, for any $\left(\epsilon, \epsilon^{\prime}\right)$, are

$$
\Gamma_{w}^{\left(\epsilon \epsilon^{\prime}\right)}(\tau)=\pi\left[2 B_{w}(\tau) \operatorname{Tr}\left(\mathcal{D}_{1}^{(\epsilon)} \mathcal{D}_{2}^{\left(\epsilon^{\prime}\right)}\right)+4 D_{w}(\tau) \operatorname{Tr}\left(\mathcal{S} \mathcal{D}_{1}^{(\epsilon)} \mathcal{D}_{2}^{\left(\epsilon^{\prime}\right)}\right)+E_{w}(\tau) \operatorname{Tr}\left(\mathcal{S} \mathcal{D}_{1}^{(\epsilon)}\right) \operatorname{Tr}\left(\mathcal{S} D_{2}^{\left(\epsilon^{\prime}\right)}\right)\right]
$$

So, the functions to be inserted in the SNR in order to take into account all the cross correlations between the two spheres are

$$
\Gamma_{w}(\tau)=\sqrt{\sum_{\epsilon=\epsilon^{\prime}}\left[\Gamma_{w}^{\left(\epsilon \epsilon^{\prime}\right)}(\tau)\right]^{2}+\frac{1}{2} \sum_{\epsilon \neq \epsilon^{\prime}}\left[\Gamma_{w}^{\left(\epsilon \epsilon^{\prime}\right)}(\tau)\right]^{2}} .
$$

In Fig 2 we compare the plots of $\Gamma_{h}(f)$ and $\Gamma_{\xi}(f)$. The former was computed in Ref. [34]. We choose $d=400 \mathrm{~km}$, the distance between the sites of AURIGA and NAUTILUS.

FIG. 2. Correlation between the quadrupole modes of two spheres, one located at the site of AURIGA (45.35 N, 11.95 E) and the other at that of NAUTILUS $(41.80 \mathrm{~N}, 12.67 \mathrm{E}), d=400 \mathrm{~km}$ : comparison between $\Gamma_{h}(f)\left(\right.$ solid line), and $\Gamma_{\xi}(f)($ dashed line).

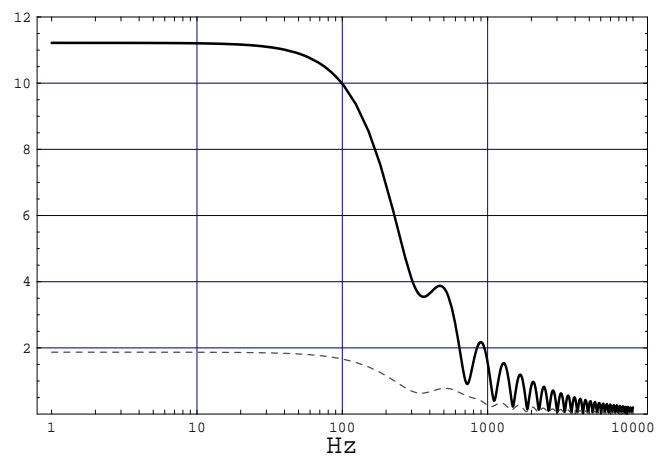

\section{Sensitivity of VIRGO with a resonant sphere}

In this subsection we evaluate the minimum detectable scalar spectrum correlating the monopole mode of a sphere and an interferometer, with the noise power spectrum of VIRGO. We label $\mathcal{D}_{1}$ the tensor of the monopole mode of the sphere and $\mathcal{D}_{2}$ the one of the interferometer. Since $\operatorname{Tr}\left(\mathcal{D}_{1}\right)=3 / 2$ and $\mathcal{D}_{2}$ is traceless, the general expression for the overlap reduction function is

$$
\Gamma_{\xi}(\tau)=\frac{\pi}{2}\left[3 C_{\xi}(\tau)+4 D_{\xi}(\tau)+E_{\xi}(\tau)\right] \operatorname{Tr}\left(\mathcal{S} \mathcal{D}_{2}\right)
$$


Explicit evaluation shows the overlap to be

$$
\Gamma_{\xi}(f)=4 \pi j_{2}(\tau) \operatorname{Tr}\left(\mathcal{S} \mathcal{D}_{2}\right) .
$$

As pointed out in Ref. [9], this function is a product of a part depending only on the distance $d$ and on the frequency and a part depending on the relative position and orientation of the detector frames. For an explicit estimate of the minimum detectable scalar spectrum we need the expression of the interferometer detector tensor with respect to the Earth centered reference frame, so that (4.30) writes in terms of the latitude and longitude of the antennas. The interferometer tensor is

$$
\mathcal{D}_{2}^{i j}=\frac{1}{4}\left[(\cos 2 \chi-\cos 2 \psi) e_{+}^{i j}(\hat{r})+(\sin 2 \chi-\sin 2 \psi) e_{\times}^{i j}(\hat{r})\right],
$$

where $\chi$ and $\psi$ are the orientations of the two interferometer arms measured counterclockwise from the true North. Therefore, the location dependence of this tensor is split into a part depending on the position of the interferometer on the Earth surface and a part depending on the orientations of the arms with respect to the true North .

Since the sphere noise power spectrum is narrowbanded with respect to that of an interferometer, we assume the latter to be constant, and equal to the value of (4.21) for $f=f_{n 0}$, within the sphere bandwidth $\Delta f_{n 0}$. This implies that the SNR can be written as

$$
\mathrm{SNR}_{\xi}=\frac{\alpha_{0}^{2}}{\left(1+\alpha_{0}^{2}\right)} \frac{3 H_{0}^{2}}{8 \pi^{3}} \sqrt{\frac{\pi}{2} \Delta_{f_{n 0}} T} \frac{\Omega_{\xi}\left(f_{n 0}\right) \Gamma_{\xi}\left(f_{n 0}\right)}{f_{n 0}^{3} \sqrt{S_{n}^{(1)}\left(f_{n 0}\right) S_{n}^{(2)}\left(f_{n 0}\right)}} .
$$

In Fig. 3 we plot $\Gamma_{\xi}(f)$ for $d=58 \mathrm{~km}$ and $d=270 \mathrm{~km}$, which is the distance between VIRGO and the site of NAUTILUS in Frascati. In this figure we plot also the curve for the case in which the sphere is located nearly in the Gran Sasso underground laboratory $(d=294 \mathrm{~km})$. For this correlation $\Gamma_{\xi}(f)$ gets approximately its maximum value. This result is particularly important in view of the remark that for the future resonant detectors with project sensitivity approaching the quantum limit, the cosmic ray interactions in the detector may set a limit to the sensitivity in an unshielded environment.

FIG. 3. The overlap reduction function $\Gamma_{\xi}$ of VIRGO (43.63 N, $10.50 \mathrm{E} ; \chi=71.5 \mathrm{deg}, \psi=341.5$ deg) with a resonant sphere located at: Frascati (41.80 N, $12.67 \mathrm{E}), d=270 \mathrm{~km}$ (solid line); (43.2 N, $10.9 \mathrm{E}), d=58 \mathrm{~km}$ (dashed line); Gran Sasso laboratory $(42.4 \mathrm{~N}, 13.70 \mathrm{E}), d=294 \mathrm{~km}$ (dotted line). See the text for further explanations.

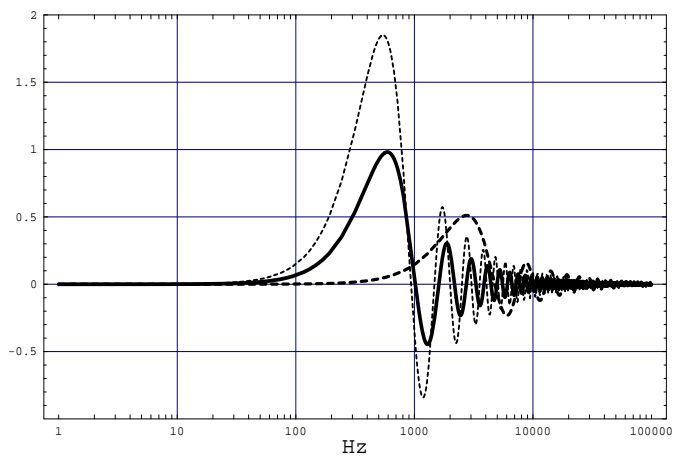

For $d=270 \mathrm{~km}$ the function has its first peak at $\simeq 591 \mathrm{~Hz}$, with $\Gamma_{\xi} \sim 0.98$, and its first zero at $\simeq 1019 \mathrm{~Hz}$. Notice that, as the spacing $d$ increases, the peak frequency moves to lower values. This is peculiar of the correlation between a sphere and an interferometer, because for two spheres, two interferometers or two bars an increase of $d$ simply implies a shift to lower values of the first zero. For a sphere and an interferometer this effect is due to the function $j_{2}(\tau)$ in (4.30). The relative orientation dependent factor, $\operatorname{Tr}\left(\mathcal{S} \mathcal{D}_{2}\right)$, accounts for the full amplitude of the overlap reduction function, being an oscillating function. In fact we can express the overlap reduction function with respect to the natural frame of the interferometer defined before [9]. In this frame, the direction of the unit vector $\hat{s}$ joining

\footnotetext{
${ }^{7}$ Notice that the computations of Ref. [9] were performed in the interferometer frame and not in the Earth centered one.
} 
the antennas is determined by the angles $(\theta, \phi)$ and the detector tensor has the simple form (3.2). Using the same convention for the angles as in [9] we have

$$
\operatorname{Tr}\left(\mathcal{S D}_{2}\right)=\frac{1}{2} \sin ^{2} \theta \cos 2 \phi .
$$

In Table $\mathrm{V}$ we consider Mo and $\mathrm{CuAl}$ hollow spheres at $d=270 \mathrm{~km}$. The $\mathrm{CuAl}$ sphere gives the best sensitivities, because the resonance frequency is lower and it belongs to the range where the overlap reduction function gets its maximum. $S_{n}^{(1)}$ is the noise power spectrum of the sphere and $S_{n}^{(2)}$ that of VIRGO, both for the frequency $f_{10} \equiv f_{0}$.

TABLE V. Minimum detectable scalar spectrum $\left(\mathrm{SNR}_{\xi}=1, T=1\right.$ year $)$ obtained by the first monopole vibrational mode of one hollow sphere at Frascati with VIRGO $(d=270 \mathrm{~km})$. The CuAl and Mo hollow spheres are those of Table II and Table III, therefore only their resonance frequencies and the corresponding sensitivities are written here.

\begin{tabular}{ccccccc}
\hline \hline & $f_{0}(\mathrm{~Hz})$ & $\Delta_{f_{0}}(\mathrm{~Hz})$ & $\Gamma_{\xi}\left(f_{0}\right)$ & $\sqrt{S_{n}^{(1)}}\left(\mathrm{Hz}^{1 / 2}\right)$ & $\sqrt{S_{n}^{(2)}}\left(\mathrm{Hz}^{1 / 2}\right)$ & $h_{0}^{2} \Omega_{\gamma_{\mathrm{Edd}}}$ \\
\hline CuAl & 770 & 24.3 & 0.76 & $2.2 \times 10^{-24}$ & $4.1 \times 10^{-23}$ & $1.2 \times 10^{-5}$ \\
& 609 & 19.2 & 0.98 & $2.9 \times 10^{-24}$ & $3.9 \times 10^{-23}$ & $6.6 \times 10^{-6}$ \\
& 498 & 15.7 & 0.92 & $3.8 \times 10^{-24}$ & $3.9 \times 10^{-23}$ & $5.6 \times 10^{-6}$ \\
& 455 & 14.4 & 0.85 & $5.6 \times 10^{-24}$ & $4.0 \times 10^{-23}$ & $7.2 \times 10^{-6}$ \\
\hline \multirow{2}{*}{$\mathrm{Mo}$} & 3027 & 95.7 & 0.19 & $9.4 \times 10^{-24}$ & $1.1 \times 10^{-22}$ & $1.8 \times 10^{-2}$ \\
& 2304 & 72.9 & 0.16 & $1.2 \times 10^{-23}$ & $8.6 \times 10^{-23}$ & $1.0 \times 10^{-2}$ \\
& 1650 & 52.2 & 0.08 & $1.2 \times 10^{-23}$ & $6.4 \times 10^{-23}$ & $7.0 \times 10^{-3}$ \\
\hline \hline
\end{tabular}

\section{CONCLUSIONS}

The aim of this paper was to study the detectability of scalar GWs from the cosmic stochastic GW background. Before discussing our results, we would briefly like to recall what is the best strategy to perform such a measurement in our opinion. As we argued in the introduction, as far as the type of detector to use is concerned, it does not seem practical to use only $\mathrm{L}$ shaped interferometers. If the impinging monopole mode of a scalar GW moves along the $\hat{z}$ axis and the arms of the interferometer lie in the $\hat{x}, \hat{y}$ plane, then the two arms will be stretched by the same quantity $\delta L$. In this case an interferometer set to work on a dark fringe will not detect any signal. On the contrary, in this very configuration a spin $2 \mathrm{GW}$ will give its maximum effect. This is a limiting case though. In general the direction of the impinging GW will form a certain angle with $\hat{z}$ and the perturbation due to a scalar will be tangled with that of the spin 2 in an inextricable way. In principle one could reconstruct the directional sensitivity patterns for the two spins to separate the two signals. The data needed to do this will require much time to be gathered and for this reason also this proposal does not seem practical to us. What would happen if the detector were a sphere? Let us first analyze the case of a GW from the viewpoint of a reference frame centered in the origin of the sphere. As it was discussed in Ref. 22 the GW interacts with the resonant mass detector through the so called electric tensor $E_{i j}=R_{i 0 j 0}$. If the direction of propagation of the incoming GW is along the $\hat{z}$ axis, the six components of this tensor can be expressed, using a null tetrad, in terms of the so called Newman-Penrose parameters which can also be expressed in the basis given by the $\mathcal{S}_{i j}^{(l m)}$ defined in Appendix C1. In turn this basis can be put in relation with the actual measurements performed by the detectors [22. In this reference frame there is a direct correspondence between the Newman-Penrose parameters and the spin of the incoming GW and the information about the spin of the GW can be easily extracted. If the direction of propagation of the GW makes an angle with the $\hat{z}$ axis, the situation is more complicated as it can also be seen from (3.14) which is the cross section given by a GW with polarization $e^{+}, e^{\times}$and from (3.24), which is the analogous with polarization $e^{s}$. In (3.12), for example, even if the incoming wave is pure spin 2 all the modes are excited and the situation is indistinguishable from that described by (3.24). The situation resembles very much that for an interferometer. But in the case of the sphere, the pure monopole mode given from (3.20) is only excited by the scalar wave, giving a clear signal of the presence of a scalar wave. This is the motivation for our proposal to couple an interferometer to a resonant detector of spherical shape. Let us now look at the results we have obtained that, for the sake of generality, encompass also the sensitivities for pairs of resonant mass detectors. 
The behavior of the overlap reduction function for pairs of resonant mass detectors (Fig. 1.2) is quite different from the one for a resonant mass detector and an interferometer (Fig. 3). In Fig. 새 2 we see a constant function which abruptly goes to zero for certain values of the frequency. These values of the frequency decrease by increasing the distance $d$ at which the two detectors are located. The values of the monopole overlap reduction function and of $\Gamma_{h}(f)$ are of the same order of magnitude: however, the quadrupole $\Gamma_{\xi}(f)$ is even an order of magnitude smaller at low frequencies.

Quite on the contrary, the overlap reduction function for the pair interferometer-resonant mass detector is different from zero only in a certain region which depends from the distance between the antennas and the direction $\hat{s}$ of the sphere with respect to the arms of the interferometers. In Fig. 3 it is shown that the values of the frequencies at which the overlap is maximum are in agreement with the resonant frequencies of the planned detectors [3].

The numerical results concerning the sensitivities are given in Tables II], III, IV for pairs of resonant mass detectors and in Table $\mathrm{V}$ for the pair interferometer-resonant mass detector.

The values given in Tables II. III show the potential of hollow spheres: going from realistic weights for such detectors of the order of the dozens of tons to weights of the order of the hundreds of tons (which are non realistic at the present state of the art) there is a gain in sensitivity of two orders of magnitude. Such a gain could also be achieved going to materials with a higher speed of sound propagation [35] as can be seen from (4.18) where both $M$ and $v$ appear in the denominator but $v$ is squared.

How do these results compare to those obtained for spin $2 \mathrm{GW}$ ? It depends on the value of the scalar amplitude $\alpha_{0}^{2}$ and the scalar coupling $\tilde{q}^{a}$ since $\Omega_{\xi}$ is roughly proportional to the scalar amplitude and coupling while $\Omega_{h}$ is roughly proportional to $1+\alpha_{0}^{2}$ (see $(4.12)$ and $\left.(4.13)\right)$. At the moment $\alpha_{0}^{2}$ has been only measured in our solar system and its value at $1 \sigma$ level is $\alpha_{0}^{2} \approx 10^{-3}[33$. Such a small value is given to the fact that Einsteinian general relativity seems to be very well verified. Such a value of $\alpha_{0}^{2}$ would give very little chances to the planned resonant detectors to detect a scalar GW background which should be limited by nucleosynthesis to be [36]

$$
\int_{f>10^{-8} H z} h_{0}^{2} \Omega_{\xi}(f) \mathrm{d}(\ln f)<10^{-5} .
$$

We have also to mention Ref. [37], where an estimate of $\alpha_{0}^{2}$ was attempted starting from the same nucleosynthesis bound: the result is a weak dependence of $\alpha_{0}^{2}$ from distance. Our ignorance on the mechanisms that should give $\alpha_{0}^{2}$ its value (cosmological attractor? supersymmetry breaking?) prevents us from further comments on this point. Also concocting strategies with resonant mass detectors made of different materials (to exploit the $\tilde{q}_{a}$ dependence) is possible, but probably premature given what we said earlier. We remark however that once operating, resonant mass detectors of spherical shape could themselves provide a measure of $\alpha_{0}^{2}$ using binary or collapsing stars as emphasized by many authors and more in particular in Ref. [25].

As a final comment we remark that the sensitivity of the pair interferometer-resonant mass detector seems to be a couple of orders of magnitude less than that of a pair of resonant mass detectors. The plots we have given show that a careful choice of where to locate the detectors can account for up to an order of magnitude in sensitivity.

\section{ACKNOWLEDGMENTS}

We would like to thank M. Gasperini for useful discussions. A.N. would like to thank Andrea Nagar for very helpful suggestions and Bobet Klein for many encouragements during the completion of this work. 


\section{APPENDIX A: CORRELATION FUNCTIONS FOR MANY SCALAR FIELDS}

The SNR for scalar GWs has been computed in Ref. [9] following Ref. [30]. Actually, although the generalization to single scalar theories (Brans-Dicke) is trivial, the one to multi scalar theory needs the introduction of a very strong constraint on the fields themselves: in order to get a formula for the SNR one can state the following lowest order condition for the correlation function between the Fourier amplitudes of the scalar fields

$$
<\xi^{a *}(f, \hat{\Omega}) \xi^{b}\left(f^{\prime}, \hat{\Omega}\right)>=\gamma_{0}^{a b} \delta\left(f-f^{\prime}\right) \delta\left(\hat{\Omega}-\hat{\Omega}^{\prime}\right) K(f)
$$

where $K(f)$ is a real non negative symmetric function. This hypothesis means that the correlation function is the same for every pair of scalar fields. This is not the most general situation one can imagine: in fact, because of the symmetry $a \leftrightarrow b$, one would expect to have $n(n+1) / 2$ distinct correlation functions $K_{a b}(f)$. On the other hand, if we consider only one degenerate function $K(f)$, then the framework is exactly the same of Refs. [9. 30], and, with the same algebra, we get (4.12).

We reproduce the main steps to express $K(f)$ in terms of the spectrum $\Omega_{s}$. Straightforward generalization of [9] shows that, for any tensor multi scalar theory, the energy density carried by a GW is

$$
\tau_{00}=\rho_{h}+\rho_{s}=\left(1+\alpha_{0}^{2}\right)(32 \pi \tilde{G})^{-1}\left[<\dot{h}_{\mu \nu} \dot{h}^{\mu \nu}>+8 \gamma_{a b}^{0}<\dot{\xi}^{a} \dot{\xi}^{b}>\right]
$$

where the brackets $\langle\ldots\rangle$ stand for integration over a finite region of tridimensional space containing several wavelengths. From this formula we recover $K(f)$ as a function of the scalar spectrum $\Omega_{s}(f)$. From (A1) one obtains

$$
<\dot{\xi}^{a} \dot{\xi}^{b}>=32 \pi^{3} \gamma_{0}^{a b} \int_{0}^{\infty} d f f^{2} K(f)
$$

Using the definition of $\Omega_{s}$, for non negative $f$, we get

$$
\Omega_{s}(f)=\frac{f}{\rho_{c}} \frac{\mathrm{d} \rho_{s}}{\mathrm{~d} f}=n\left(1+\alpha_{0}^{2}\right) \frac{64 \pi^{3}}{3 H_{0}^{2}} f^{3} K(f),
$$

where we used $\gamma_{a b}^{0} \gamma_{0}^{a b}=n$. Furthermore $\Omega_{s}=\sum_{a} \Omega_{\xi^{a}}=n \Omega_{\xi^{a}}$ so we can infer from (A4)

$$
K(f)=\left(1+\alpha_{0}^{2}\right)^{-1} \frac{3 H_{0}^{2}}{64 \pi^{3}} f^{-3} \Omega_{\xi}(f),
$$

where $\Omega_{\xi^{a}} \equiv \Omega_{\xi}$.

\section{APPENDIX B: THE OVERLAP REDUCTION FUNCTIONS}

In the following we give the coefficients, introduced in the main text, for the functions $\Gamma_{\xi}(f)$

$$
\left(\begin{array}{l}
A \\
B \\
C \\
D \\
E
\end{array}\right)_{\xi}=\frac{4}{\tau^{2}}\left(\begin{array}{c}
\tau^{2} j_{0}(\tau)-2 \tau j_{1}(\tau)+j_{2}(\tau) \\
j_{2}(\tau) \\
-\tau^{2} j_{0}(\tau)+4 \tau j_{1}(\tau)-5 j_{2}(\tau) \\
\tau j_{1}(\tau)-5 j_{2}(\tau) \\
\tau^{2} j_{0}(\tau)-10 \tau j_{1}(\tau)+35 j_{2}(\tau)
\end{array}\right)
$$

and $\Gamma_{h}(f)$

$$
\left(\begin{array}{l}
A \\
B \\
C \\
D \\
E
\end{array}\right)_{h}=\frac{4}{\tau^{2}}\left(\begin{array}{c}
-\tau^{2} j_{0}(\tau)-2 \tau j_{1}(\tau)+j_{2}(\tau) \tau^{2} j_{0}(\tau)-2 \tau j_{1}(\tau)+j_{2}(\tau) \\
-\tau^{2} j_{0}(\tau)-2 \tau j_{1}(\tau)-5 j_{2}(\tau) \\
-\tau^{2} j_{0}(\tau)+4 \tau j_{1}(\tau)-5 j_{2}(\tau) \\
\tau^{2} j_{0}(\tau)-10 \tau j_{1}(\tau)+35 j_{2}(\tau)
\end{array}\right)
$$




\section{APPENDIX C: DETECTOR TENSORS}

\section{The sphere mode tensors}

A basis for the pure spherical harmonics is given by the $\mathcal{S}^{(l m)}$, with $l=0,2$ 20

$$
\begin{aligned}
\mathcal{S}^{(00)} & \equiv \frac{1}{\sqrt{4 \pi}}\left(\begin{array}{ccc}
1 & 0 & 0 \\
0 & 1 & 0 \\
0 & 0 & 1
\end{array}\right), \\
\mathcal{S}^{(20)} & \equiv \sqrt{\frac{5}{16 \pi}}\left(\begin{array}{ccc}
-1 & 0 & 0 \\
0 & -1 & 0 \\
0 & 0 & 2
\end{array}\right), \\
\mathcal{S}^{(2 \pm 2)} & \equiv \sqrt{\frac{15}{32 \pi}}\left(\begin{array}{ccc}
1 & \pm i & 0 \\
\pm i & -1 & 0 \\
0 & 0 & 0
\end{array}\right), \\
\mathcal{S}^{(2 \pm 1)} & \equiv \sqrt{\frac{15}{32 \pi}}\left(\begin{array}{ccc}
0 & 0 & \mp 1 \\
0 & 0 & -i \\
\mp 1 & -i & 0
\end{array}\right) .
\end{aligned}
$$

The normalization is chosen so that $\mathcal{S}_{i j}^{(l m)} \hat{n}^{i} \hat{n}^{j}=Y_{l m} . \hat{n}$ is the radial unit vector. The vibrational response of a spherical detector is usually written in terms of this pure spin basis. Otherwise, following Zhou and Michelson [19], the vibrations of a resonant sphere are more conveniently described as functions of the real quadrupole spherical harmonics, in addition to the monopole spherical harmonic $Y_{00}=(4 \pi)^{-1 / 2}$

$$
\begin{aligned}
Y_{0} & \equiv Y_{20}, \\
Y_{1 c} & \equiv \frac{1}{\sqrt{2}}\left(Y_{2-1}-Y_{2+1}\right), \\
Y_{1 s} & \equiv \frac{i}{\sqrt{2}}\left(Y_{2-1}+Y_{2+1}\right), \\
Y_{2 c} & \equiv \frac{1}{\sqrt{2}}\left(Y_{2-2}+Y_{2+2}\right), \\
Y_{2 s} & \equiv \frac{i}{\sqrt{2}}\left(Y_{2-2}-Y_{2+2}\right) .
\end{aligned}
$$

A convenient basis for the real spherical harmonics is given by $\mathcal{D}^{(00)} \equiv \sqrt{\pi} \mathcal{S}^{(00)}$ and $\mathcal{D}^{(\epsilon)}$ with $\epsilon \equiv 0,1 c, 1 s, 2 c, 2 s$. These traceless tensors are defined as

$$
\begin{aligned}
\mathcal{D}^{(0)} & \equiv \frac{\sqrt{3}}{6}\left(\begin{array}{ccc}
1 & 0 & 0 \\
0 & 1 & 0 \\
0 & 0 & -2
\end{array}\right), \\
\mathcal{D}^{(1 c)} & \equiv-\frac{1}{2}\left(\begin{array}{ccc}
0 & 0 & 1 \\
0 & 0 & 0 \\
1 & 0 & 0
\end{array}\right) \\
\mathcal{D}^{(1 s)} & \equiv-\frac{1}{2}\left(\begin{array}{ccc}
0 & 0 & 0 \\
0 & 0 & 1 \\
0 & 1 & 0
\end{array}\right) \\
\mathcal{D}^{(2 c)} & \equiv \frac{1}{2}\left(\begin{array}{ccc}
1 & 0 & 0 \\
0 & -1 & 0 \\
0 & 0 & 0
\end{array}\right) \\
\mathcal{D}^{(2 s)} & \equiv-\frac{1}{2}\left(\begin{array}{ccc}
0 & 1 & 0 \\
1 & 0 & 0 \\
0 & 0 & 0
\end{array}\right)
\end{aligned}
$$

with

$$
\sum_{i} \sum_{j} \mathcal{D}_{i j}^{(\epsilon)} \mathcal{D}_{i j}^{\left(\epsilon^{\prime}\right)}=\frac{1}{2} \delta^{\epsilon \epsilon^{\prime}}
$$


From the definition of the real spherical harmonics we have

$$
\begin{aligned}
\mathcal{D}^{(0)} & =-\sqrt{\frac{4 \pi}{15}} \mathcal{S}^{(20)}, \\
\mathcal{D}^{(1 c)} & =\sqrt{\frac{2 \pi}{15}}\left(\mathcal{S}^{(2+1)}-\mathcal{S}^{(2-1)}\right), \\
\mathcal{D}^{(1 s)} & =-i \sqrt{\frac{2 \pi}{15}}\left(\mathcal{S}^{(2+1)}+\mathcal{S}^{(2-1)}\right), \\
\mathcal{D}^{(2 c)} & =\sqrt{\frac{2 \pi}{15}}\left(\mathcal{S}^{(2+2)}+\mathcal{S}^{(2-2)}\right), \\
\mathcal{D}^{(2 s)} & =i \sqrt{\frac{2 \pi}{15}}\left(\mathcal{S}^{(2+2)}-\mathcal{S}^{(2-2)}\right) .
\end{aligned}
$$

\section{Explicit expressions of the polarization tensors in the detector frame}

Let us consider now the wave frame $(\hat{m}, \hat{n}, \hat{\Omega})$ and the detector frame $(\hat{x}, \hat{y}, \hat{z})$ defined in (3.5) by introducing the rotation matrix

$$
R(\hat{\Omega}) \equiv\left(\begin{array}{ccc}
\cos \phi & \sin \phi & 0 \\
-\cos \theta \sin \phi & \cos \theta \cos \phi & \sin \theta \\
\sin \theta \sin \phi & -\sin \theta \cos \phi & \cos \theta
\end{array}\right)
$$

where the angles $(\theta, \phi)$ are defined following the conventions of Forward [16]. The polarization tensors of the GW in the antenna frame $e^{B}(\hat{\Omega})$ are obtained by rotating the ones in the wave frame $e^{B}$ as

$$
e^{B}(\hat{\Omega})=R^{t}(\hat{\Omega}) e^{B} R(\hat{\Omega}) ; \quad B=\times,+, s .
$$

The tensors in the wave frame are

$$
e^{+}=\left(\begin{array}{ccc}
1 & 0 & 0 \\
0 & -1 & 0 \\
0 & 0 & 0
\end{array}\right), \quad e^{\times}=\left(\begin{array}{ccc}
0 & 1 & 0 \\
1 & 0 & 0 \\
0 & 0 & 0
\end{array}\right), \quad e^{s}=\left(\begin{array}{lll}
1 & 0 & 0 \\
0 & 1 & 0 \\
0 & 0 & 0
\end{array}\right)
$$

From (C7), for the spin 2 polarization tensors we get

$$
\begin{gathered}
e^{+}(\hat{\Omega})=\hat{m} \otimes \hat{m}-\hat{n} \otimes \hat{n}=\frac{1}{2}\left(\begin{array}{ccc}
2\left(\cos ^{2} \phi-\cos ^{2} \theta \sin ^{2} \phi\right) & \left(1+\cos ^{2} \theta\right) \sin 2 \phi & \sin 2 \theta \sin \phi \\
\left(1+\cos ^{2} \theta\right) \sin 2 \phi & 2\left(\sin ^{2} \phi-\cos ^{2} \theta \cos ^{2} \phi\right) & -\sin 2 \theta \cos \phi \\
\sin 2 \theta \sin \phi & -\sin 2 \theta \cos \phi & -2 \sin ^{2} \theta
\end{array}\right), \\
e^{\times}(\hat{\Omega})=\hat{m} \otimes \hat{n}+\hat{n} \otimes \hat{n}=\left(\begin{array}{ccc}
-\cos \theta \sin 2 \phi & \cos \theta \cos 2 \phi & \cos \phi \sin \theta \\
\cos \theta \cos 2 \phi & \cos \theta \sin 2 \phi & \sin \theta \sin \phi \\
\cos \phi \sin \theta & \sin \theta \sin \phi & 0
\end{array}\right) .
\end{gathered}
$$

For the scalar polarization tensor we have

$$
e^{s}(\hat{\Omega})=\hat{m} \otimes \hat{m}+\hat{n} \otimes \hat{n}=\frac{1}{2}\left(\begin{array}{ccc}
2\left(\cos ^{2} \phi+\cos ^{2} \theta \sin ^{2} \phi\right) & \sin ^{2} \theta \sin 2 \phi & -\sin 2 \theta \sin \phi \\
\sin ^{2} \theta \sin 2 \phi & 2\left(\sin ^{2} \phi+\cos ^{2} \theta \cos ^{2} \phi\right) & \sin 2 \theta \cos \phi \\
-\sin 2 \theta \sin \phi & \sin 2 \theta \cos \phi & 2 \sin ^{2} \theta
\end{array}\right) .
$$

\section{The Earth centered reference frame}

In Appendix B we have listed the coefficients which give the dependence of the overlap reduction function on the frequency and on the distance between the antennas. To infer its dependence on the relative orientations of the detectors, it is convenient to express the detector tensors in the reference frame of the Earth. We then express the 
detector tensors with respect to a triad of orthogonal unit vectors $(\hat{x}, \hat{y}, \hat{r})$, where $\hat{x}$ and $\hat{y}$ lie on the tangent plane and $\hat{r}$ points along the Earth radius. This triad defines univocally the antenna coordinate system. Given the latitude, $\Theta$, measured in degrees North from the equator and the longitude, $\Phi$, in degrees East of Greenwich, England, the relation of the triad of vectors $(\hat{x}, \hat{y}, \hat{r})$ with respect to the Cartesian reference frame $(\hat{X}, \hat{Y}, \hat{Z})$ originated in the center of the Earth is

$$
\begin{aligned}
& \hat{x}=-\sin \Theta \cos \Phi \hat{X}-\sin \Theta \sin \Phi \hat{Y}+\cos \Theta \hat{Z}, \\
& \hat{y}=-\sin \Phi \hat{X}+\cos \Phi \hat{Y}, \\
& \hat{r}=\cos \Theta \cos \Phi \hat{X}+\cos \Theta \sin \Phi \hat{Y}+\sin \Theta \hat{Z}
\end{aligned}
$$

A simple example is given by the tensor of an interferometer [16] which, in the Earth centered frame, is usually written as

$$
\mathcal{D}_{i j}(\hat{X}, \hat{Y})=\frac{1}{2}\left(\hat{X}_{i} \hat{X}_{j}-\hat{Y}_{i} \hat{Y}_{j}\right)
$$

where $\hat{X}$ and $\hat{Y}$ are chosen to point in the detector arms directions.

Frame dependent expressions of the same kind can also be written for the tensors describing the geometrical features of the modes of a resonant sphere. In the Earth centered reference frame, equations (C5) become

$$
\begin{aligned}
\mathcal{D}_{i j}^{(0)} & =\frac{\sqrt{3}}{6}\left(\hat{X}_{i} \hat{X}_{j}+\hat{Y}_{i} \hat{Y}_{j}-2 \hat{Z}_{i} \hat{Z}_{j}\right), \\
\mathcal{D}_{i j}^{(1 c)} & =-\frac{1}{2}\left(\hat{X}_{i} \hat{Z}_{j}+\hat{Z}_{i} \hat{X}_{j}\right), \\
\mathcal{D}_{i j}^{(1 s)} & =-\frac{1}{2}\left(\hat{Y}_{i} \hat{Z}_{j}+\hat{Z}_{i} \hat{Y}_{j}\right), \\
\mathcal{D}_{i j}^{(2 c)} & =\frac{1}{2}\left(\hat{X}_{i} \hat{X}_{j}-\hat{Y}_{i} \hat{Y}_{j}\right), \\
\mathcal{D}_{i j}^{(2 s)} & =-\frac{1}{2}\left(\hat{X}_{i} \hat{Y}_{j}+\hat{Y}_{i} \hat{X}_{j}\right) .
\end{aligned}
$$


[1] A. Abramovici et al., Science 256, 325 (1992).

[2] B. Caron et al., Class. Quantum Grav. 14, 1461 (1997).

[3] E. Coccia, V. Fafone, and G. Frosssati, in First Edoardo Amaldi Conference in Gravitational Wave Experiments, eds. E. Coccia et al. (World Scientific, Singapore, 1995).

[4] C.M. Will, Theory and Experiment in Gravitational Physics (Cambridge University Press, Cambridge, 1993).

[5] N. Arkani-Hamed, S. Dimopoulos, and G. Dvali, Phys. Lett. B429, 263 (1998).

[6] I. Antoniadis, N. Arkani-Hamed, S. Dimopoulos, and G. Dvali, Phys. Lett. B436, 257 (1998).

[7] I. Antoniadis, S. Dimopoulos, and G. Dvali, Nucl. Phys. B516, 70 (1998).

[8] M.E. Tobar, T. Suzuki and K. Kuroda, Phys. Rev. D 59, 102002 (1999).

[9] M. Maggiore and A. Nicolis, Phys. Rev. D 62, 024004 (2000).

[10] T. Damour and G. Esposito-Farèse, Class. Quantum Grav. 9, 2093 (1992).

[11] M. Gasperini, Phys. Lett. B 470, 67 (1999).

[12] M. Gasperini, Phys. Lett. B 477, 242 (2000).

[13] M. Gasperini and C. Ungarelli, Detecting a relic background of scalar waves with LIGO, gr-qc/0103035.

[14] S. Weinberg, Gravitation and Cosmology, principle and applications of the general theory of relativity, (John Wiley, New York, 1972).

[15] P. Jordan, Nature 164, 637 (1949); M. Fierz, Helv. Phys. Acta 29, 128 (1956); P. Jordan, Z. Phys. 157, 112 (1959); C. Brans and R.H. Dicke, Phys. Rev. 124, 925 (1961); R.V. Wagoner, Phys. Rev. D 1, 3029 (1970).

[16] R.L. Forward, Phys. Rev. D 17, 379 (1978).

[17] K. Nakao, T. Harada, M. Shibata, S. Kawamura and T. Nakamura, Phys. Rev. D 63, 082001 (2001).

[18] R.V. Wagoner and H.J. Paik, in Proceedings of the International Symposium on Experimental Gravitation, Pavia 1976 (Accademia Nazionale dei Lincei, Rome, 1977).

[19] C.Z. Zhou and P.F. Michelson, Phys. Rev. D 51, 2517 (1995).

[20] J.A. Lobo, Phys. Rev. D 52, 591 (1995).

[21] E. Coccia, V. Fafone, G. Frossati, J.A. Lobo, and J.A. Ortega, Phys. Rev. D 57, 2051 (1998).

[22] M. Bianchi, E. Coccia, C.N. Colacino, V. Fafone, and F. Fucito, Class. Quantum Grav. 13, 2865 (1996).

[23] M. Bianchi, M. Brunetti, E. Coccia, F. Fucito, and J.A. Lobo, Phys. Rev. D 57, 4525 (1998).

[24] M. Brunetti, E. Coccia, V. Fafone, and F. Fucito, Phys. Rev. D 59, 044027 (1999).

[25] E. Coccia, F. Fucito, M. Salvino, and J.A. Lobo, Phys. Rev. D 62, 044019, (2000).

[26] L. Baiotti, Teorie scalar-tensoriali e Formalismo Post-Newtoniano, Tesi di Laurea, (Turin 2000, unpublished).

[27] P. Astone, G.V. Pallottino, and G. Pizzella, Class. Quantum Grav. 14, 2019 (1997).

[28] P.F. Michelson, Mon. Not. R. Astr. Soc. 227, 933 (1987); N. Christensen, Phys. Rev. D 46, 5250 (1992).

[29] E. Flanagan, Phys. Rev. D 48, 2389 (1993).

[30] B. Allen and J.D. Romano, Phys. Rev. D 59, 102001 (1999).

[31] M. Maggiore, Phys. Rep. 331, 283 (2000).

[32] E. Cuoco, G. Curci, and M. Beccaria, in Second Amaldi Conference on Gravitational Waves, eds. E. Coccia et al. (World Scientific, Singapore, 1998), preprint gr-qc/9709041.

[33] D.E. Lebach et al., Phys. Rev. Lett. 75, 1439 (1995); R.D. Reasenberg et al. Astrophys. J. 234, L219 (1979); D.S. Robertson, W.E. Carter, and W.H. Dillinger, Nature (London) 349, 768 (1991).

[34] S. Vitale, M. Cerdonio, E. Coccia, and A. Ortolan, Phys. Rev. D 55, 1741 (1997).

[35] M. Cerdonio, L. Conti, J.A. Lobo, A. Ortolan, and J.P. Zendri, Wideband dual sphere detector of gravitational waves, gr-qc/0011002.

[36] V. F. Schwartzman, Pis'ma Zh. Éksp. Teor. Fiz 9, 315 (1969) [JETP Lett 9, 184 (1969)]; T. Walker et al., Astrophys. J. 376, 51 (1991); C. Copi et al., Phys. Rev. Lett. 75, 3981 (1995); R. E. Lopez and M. S. Turner, Phys. Rev. D 59, 103502 (1999).

[37] D. I. Santiago, D. Kalligas, and R. V. Wagoner, Phys. Rev. D 56, 7627 (1997). 